\title{
The Mu2e Experiment
}

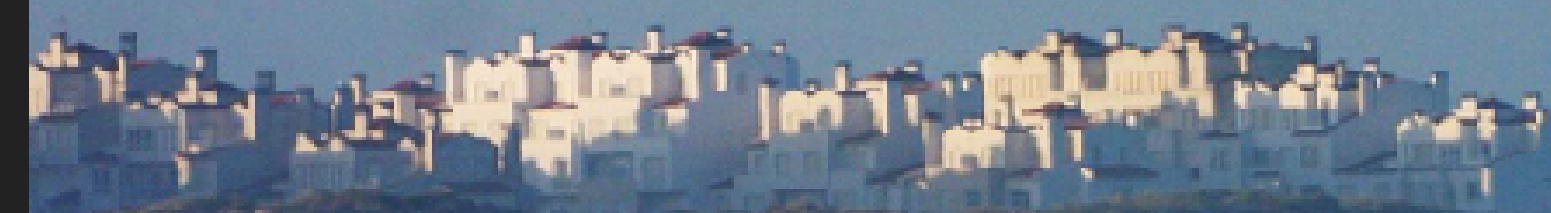

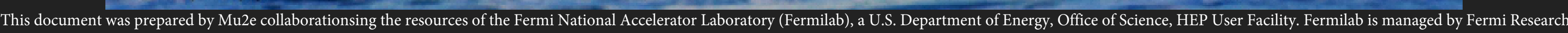
Alliance, LLC (FRA), acting under Contract No. DE-AC02-07CH11359.

\section{BEACH 2018 Peniche, Portugal}




\section{A Search for CLFV Using Muons}

o In 2021, Mu2e will search for coherent neutrinoless conversion of muon into an electron, within the vicinity of a nucleus, $\mu N \rightarrow e N$

> 3-years of data collection

$\checkmark 10,000$-fold improvement over existing limits

> Access to virtual effects on an effective mass scale of up to $10,000 \mathrm{TeV}$

Could discover CLFV

- Proving the existence of new phenomena, and their experimental accessibility

- We're complementary to other CLFV searches

- MEG, Mu3e

- Higgs decays, Tau decays, meson decays
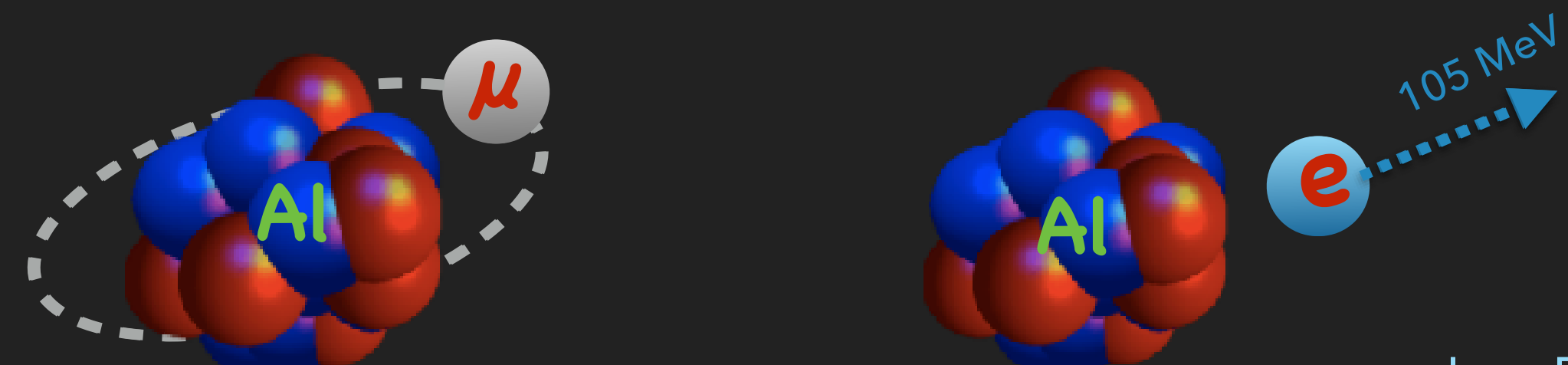


\section{Outline}

\section{Mu2e}
○ CLFV
- Experimental Details
o The Future 
- The quarks commit Flavor Violation D They mix via the $W$

\section{ELEMENTARY PARTICLES}

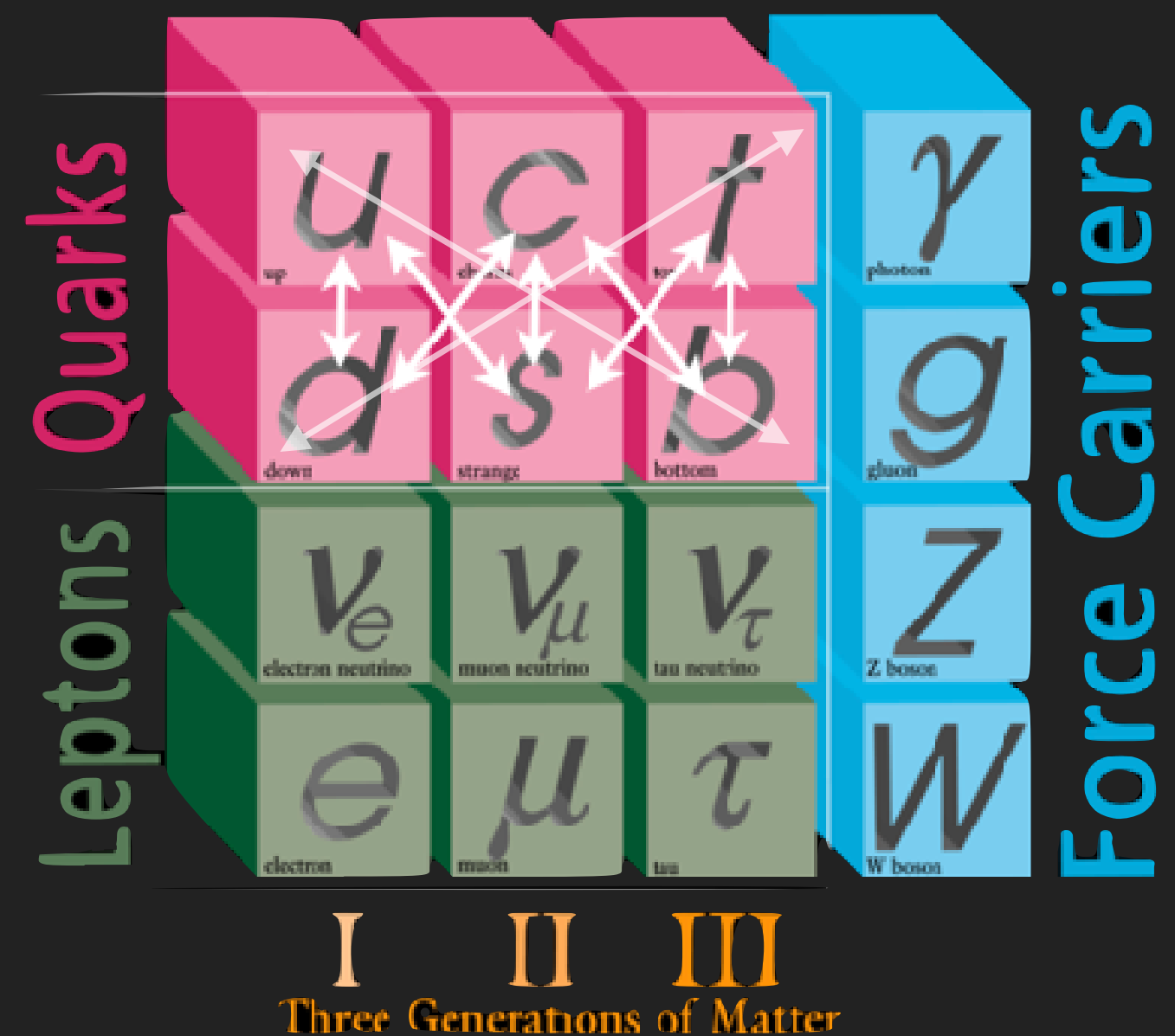




\section{Flavor Violation in the SM}

o The quarks commit Flavor Violation - They mix via the $W$

o The neutrinos can change into their partners (and vice versa)

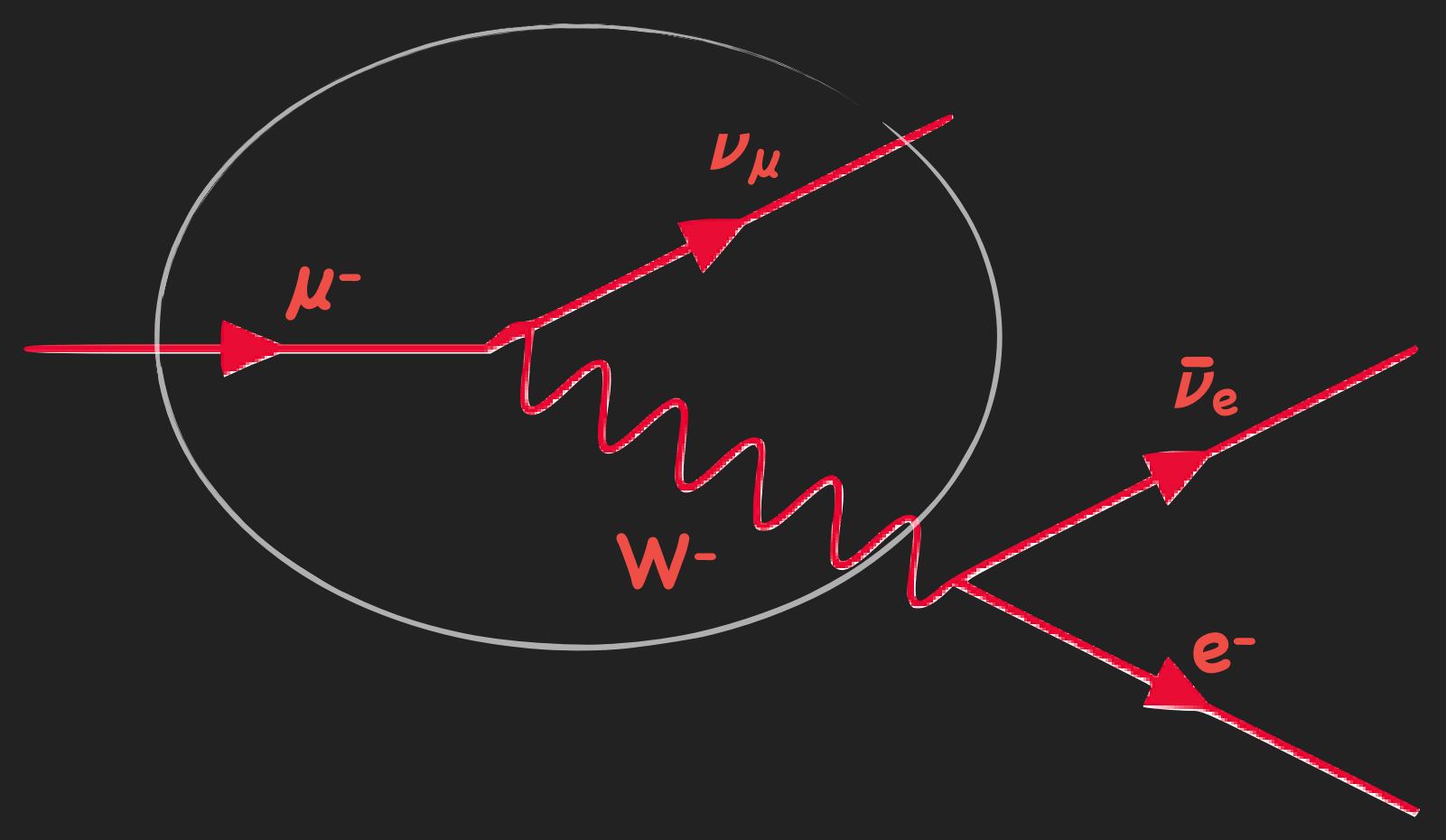

ELEMENTARY PARTICLES

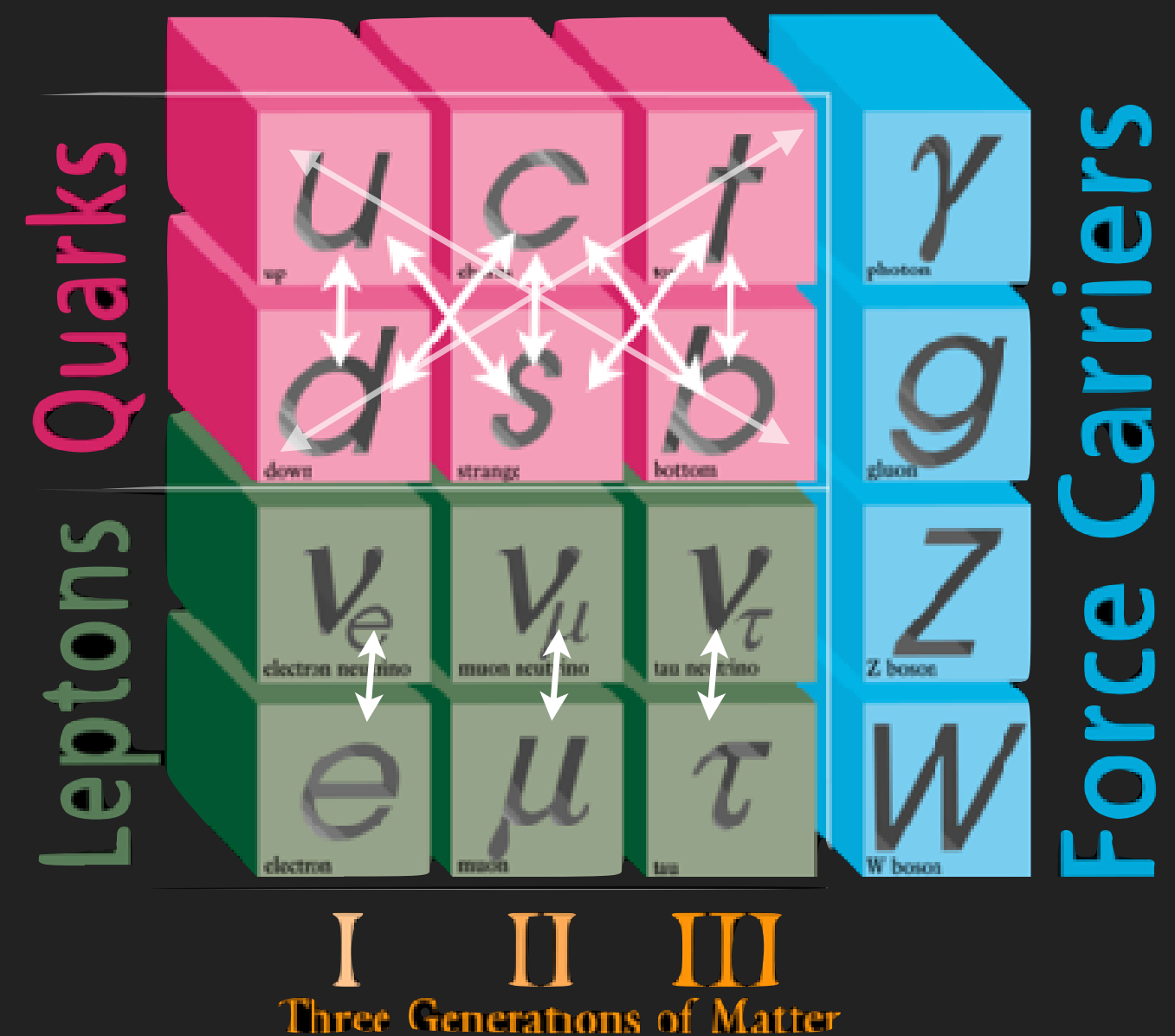




\section{Flavor Violation in the SM}

- The quarks commit Flavor Violation v They mix via the $W$

o The neutrinos can change into their partners (and vice versa)

$\circ$ The neutrinos mix

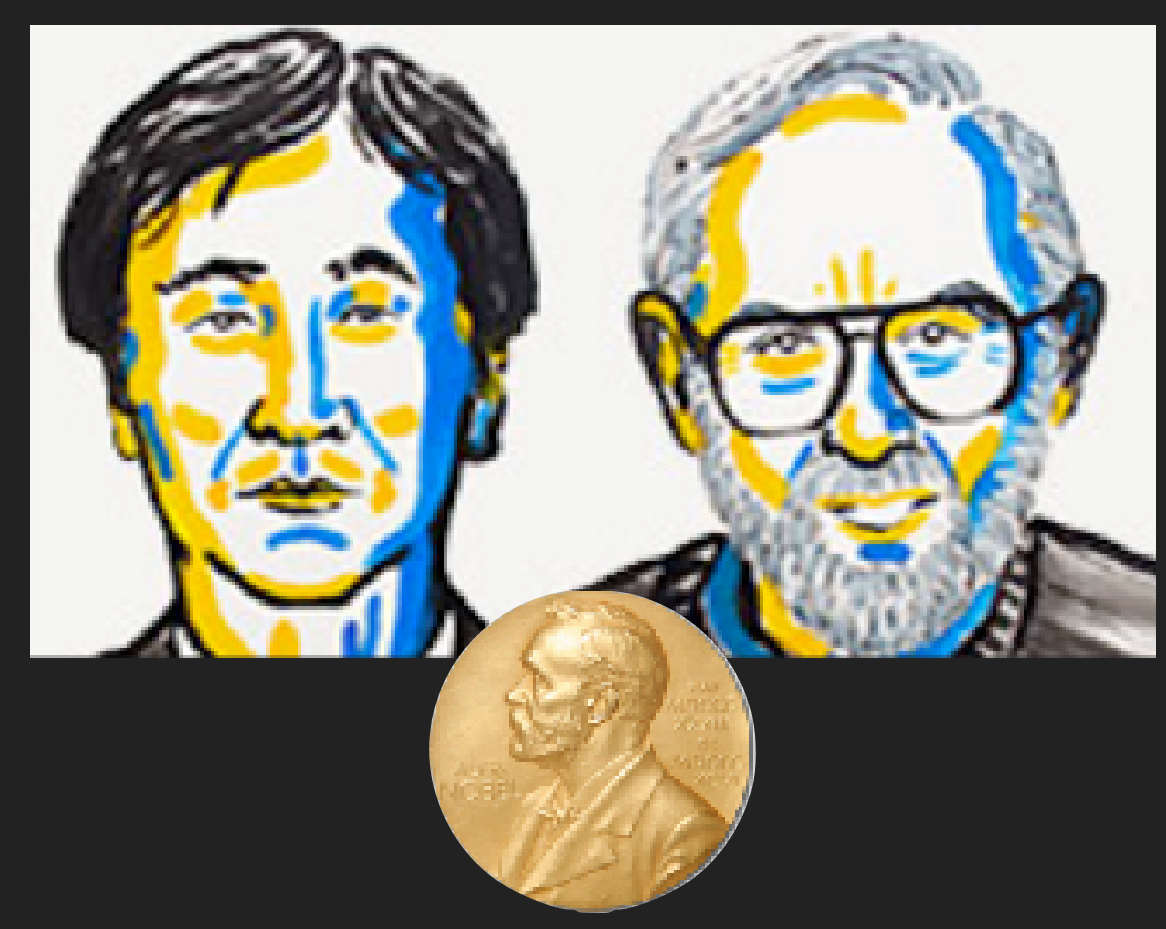

ELEMENTARY PARTICLES

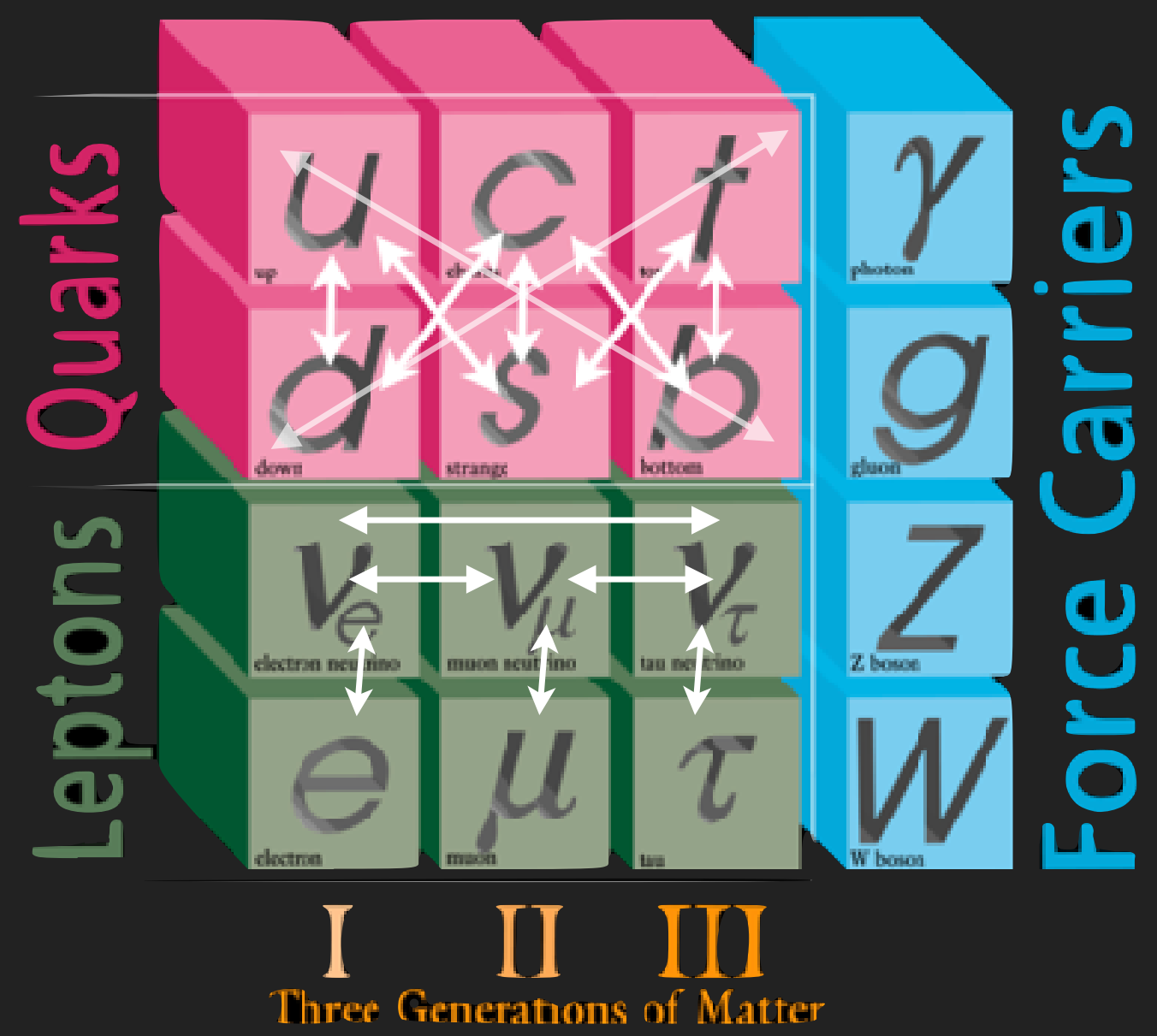




\section{Flavor Violation in the SM}

( ) The quarks commit Flavor Violation

v They mix via the $W$

- The neutrinos can change into their

partners (and vice versa)

- The neutrinos mix
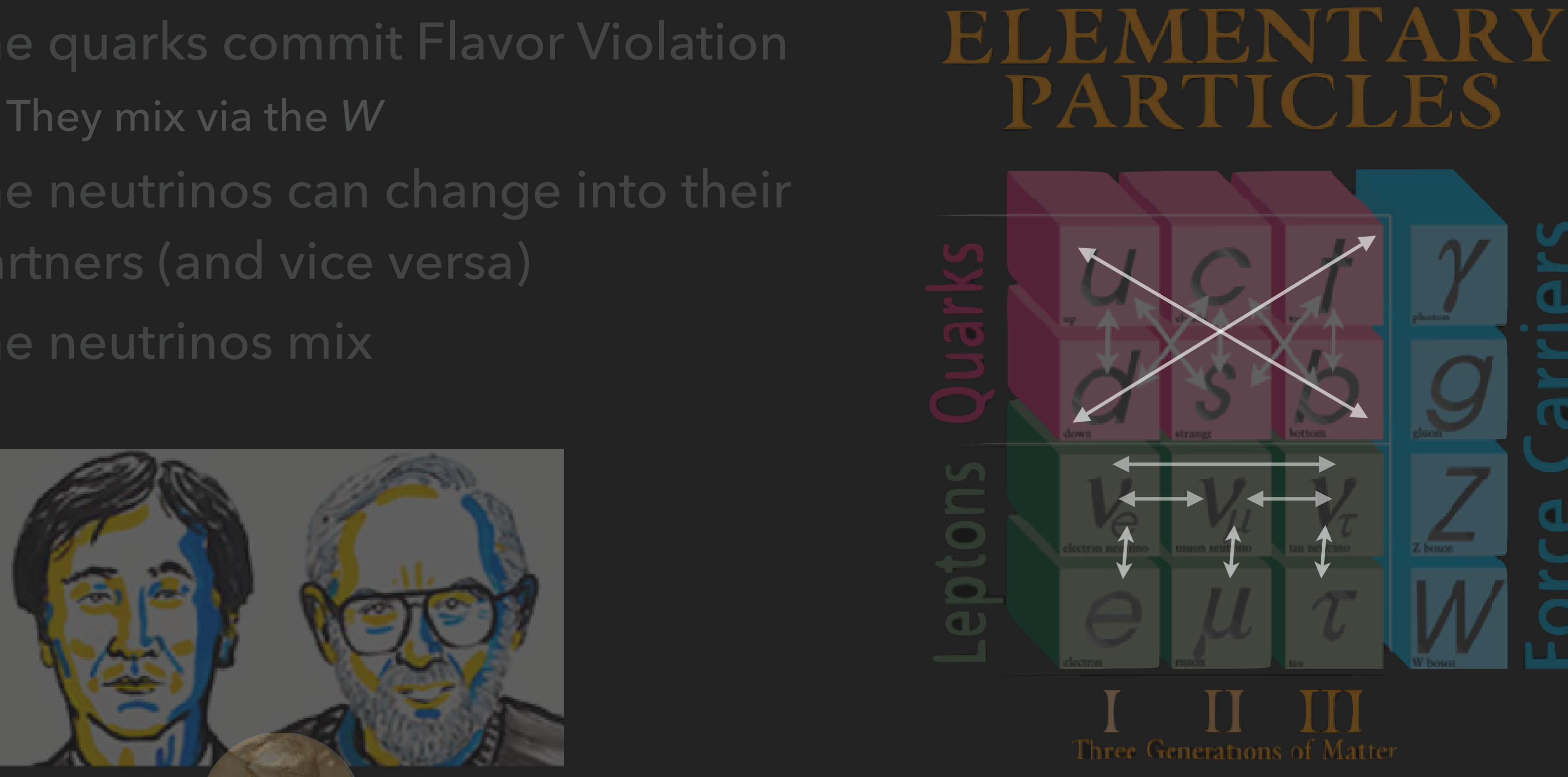

We're looking for the same thing, but with charged leptons 


\section{CLFV in the Standard Model}

- All SM CLFV rates are exactly zero

- Dynamical suppression: an accidental symmetry

○ The discovery of neutrino mixing has new implications for CLFV...

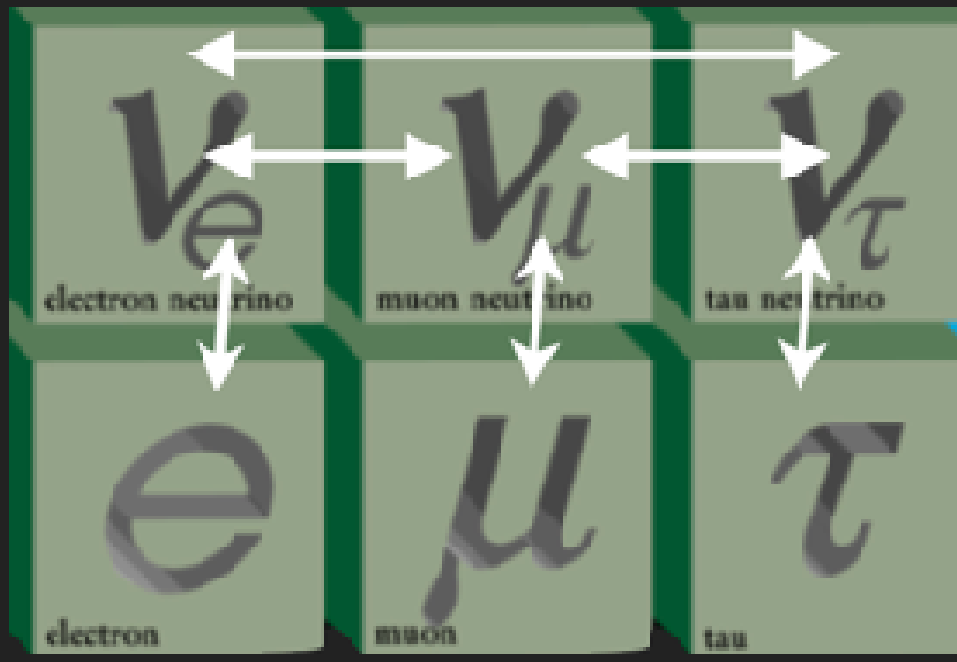




\section{CLFV in the Standard Model}

- All SM CLFV rates are exactly zero

- Dynamical suppression: an accidental symmetry

○ The discovery of neutrino mixing has new implications for CLFV...

- CLF is not an exact symmetry of our universe
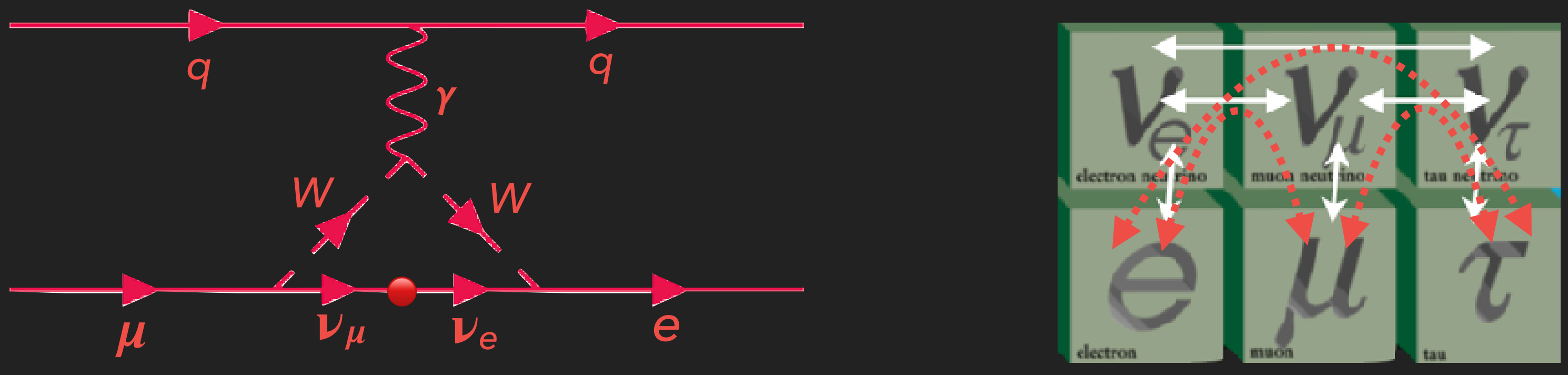


\section{CLFV in the Standard Model}

- All SM CLFV rates are exactly zero

- Dynamical suppression: an accidental symmetry

o The discovery of neutrino mixing has new implications for CLFV...

- CLF is not an exact symmetry of our universe

v But CLFV in the $\nu M S M$ is too small to see

- The observation of CLFV unambiguously indicates new physics

$$
\text { e.g. } \quad \mathcal{B}(\mu \rightarrow e \gamma)=\frac{3 \alpha}{32 \pi}\left|\sum_{i=2,3} U_{\mu i}^{*} U_{e i} \frac{\Delta m_{i l}^{2}}{M_{W}^{2}}\right|^{2} \sim 10^{-54}
$$




\section{CLFV in the Standard Model}

- All SM CLFV rates are exactly zero

- Dynamical suppression: an accidental symmetry

o The discovery of neutrino mixing has new implications for CLFV...

- CLF is not an exact symmetry of our universe

v But CLFV in the $\nu M S M$ is too small to see

- The observation of CLFV unambiguously indicates new physics

- Neutrino mass generation beyond UMSM often enhances CLFV to observable levels

$$
\text { e.g. } \quad \mathcal{B}(\mu \rightarrow e \gamma)=\frac{3 \alpha}{32 \pi}\left|\sum_{i=2,3} U_{\mu i}^{*} U_{e i} \frac{\Delta m_{i l}^{2}}{M_{W}^{2}}\right|^{2} \sim 10^{-54}
$$




\section{Magnetic moment type operator}

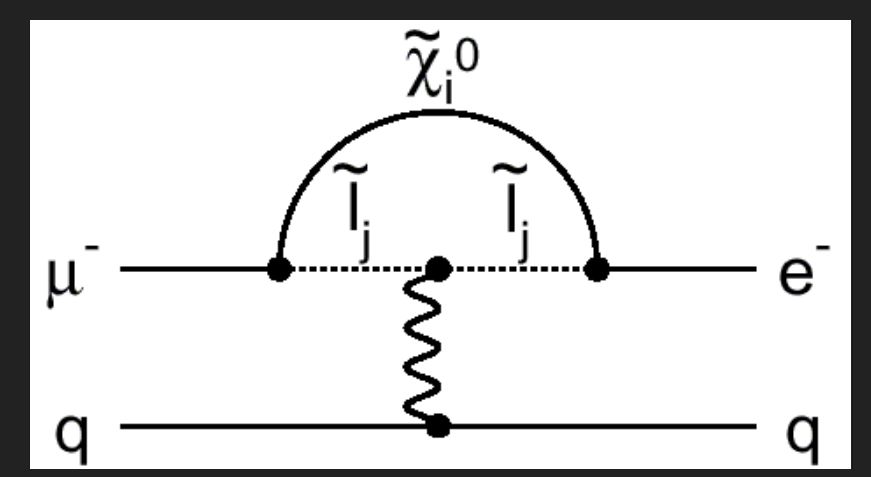

Supersymmetry

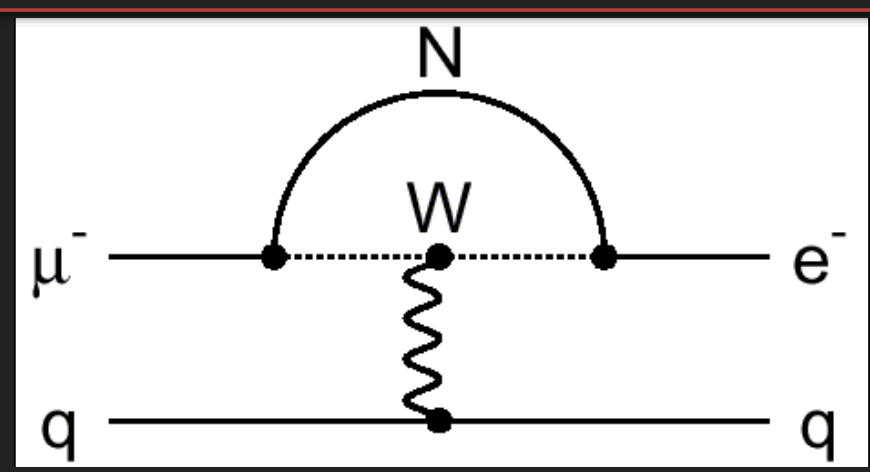

Heavy neutrinos

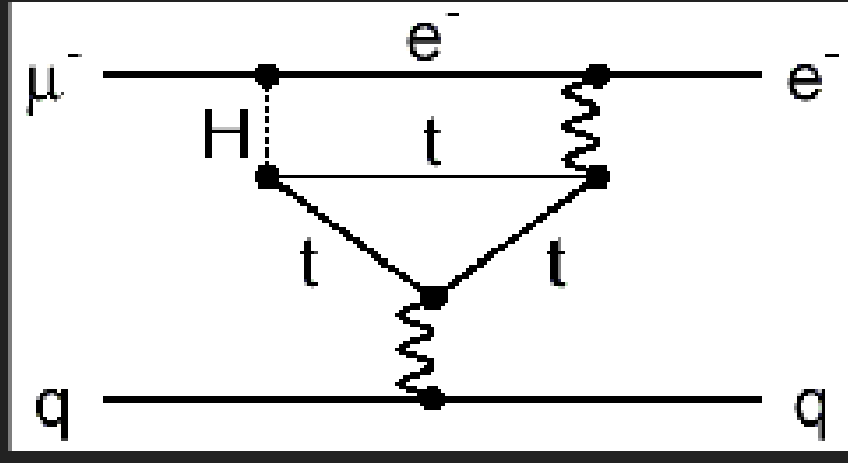

Two Higgs doublets
Contact term operator

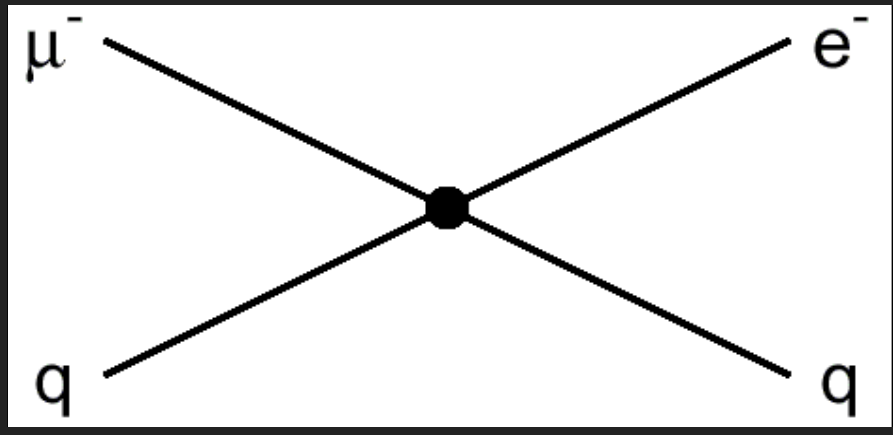

Compositeness

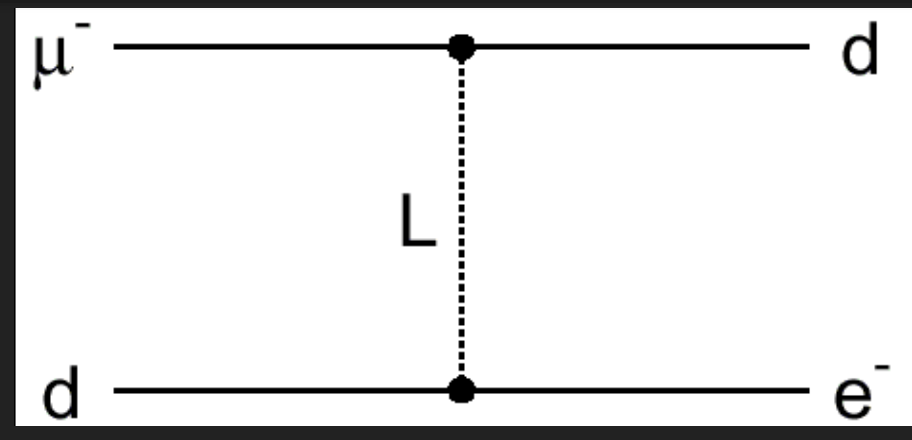

Leptoquarks

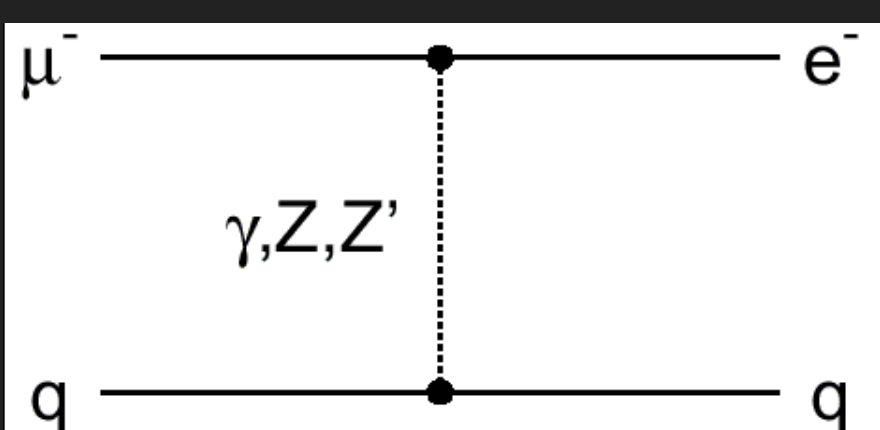

New heavy bosons / anomalous coupling 


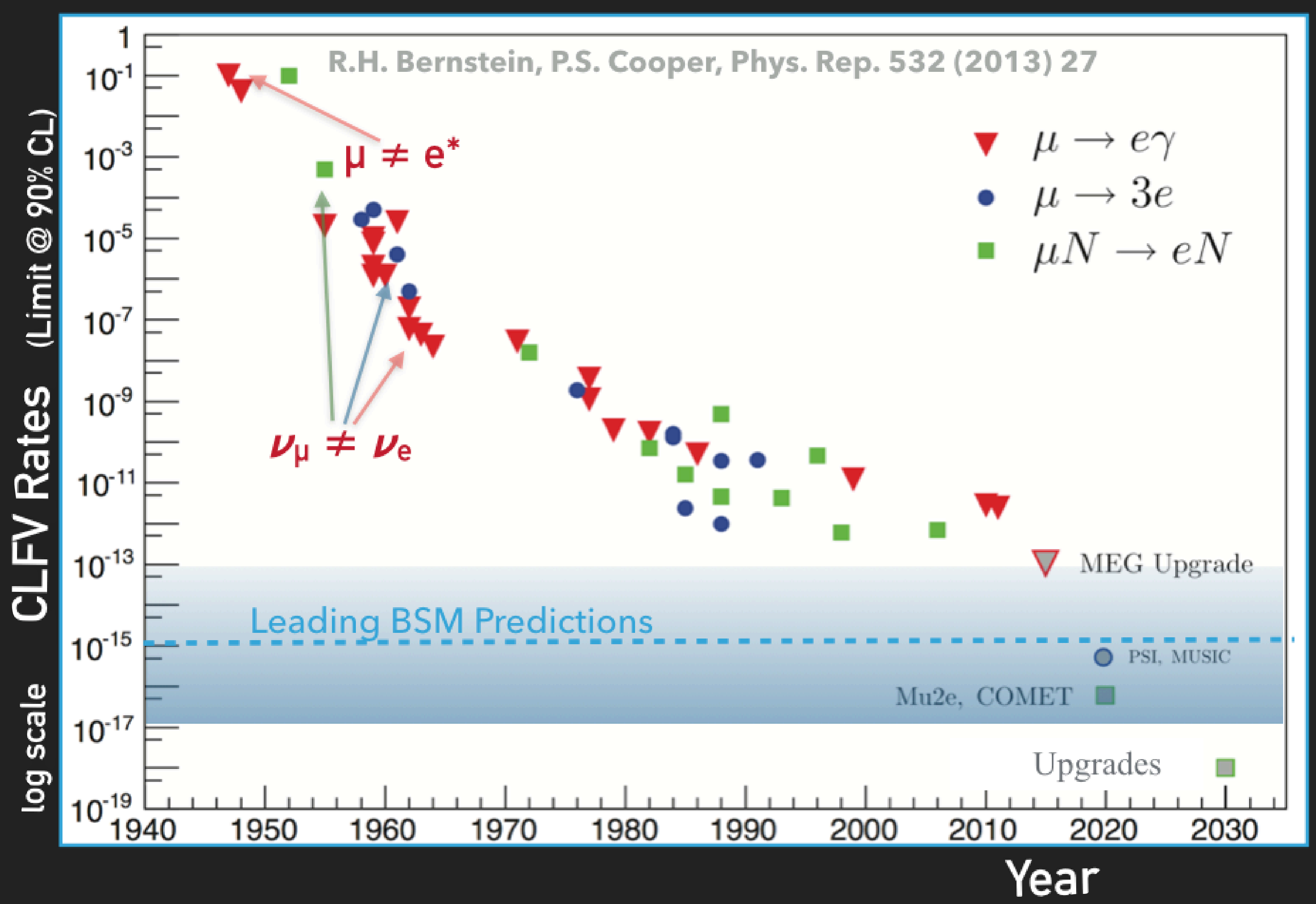




\section{Outline}

\section{Mu2e}
o ELF
- Experimental Details
o The Future 


\section{Muonic Aluminum}

- Negative muons are stopped and captured by Al

- They cascade to the $1 \mathrm{~S}$ state quickly! $\mathscr{O}(\mathrm{fs})$

- X-ray emission can give an estimate the number of captures

- Bohr radius $\sim 10 \mathrm{fm}$

- Large degree of overlap (Al nucleus $~ 3 \mathrm{fm}$ )

- Wait for a muon to convert into an electron

- Coherent process

- Mono energetic signal

- $E_{e}=m_{\mu} c^{2}-E_{b}-E_{\text {recoil }}=104.96 \mathrm{MeV}$

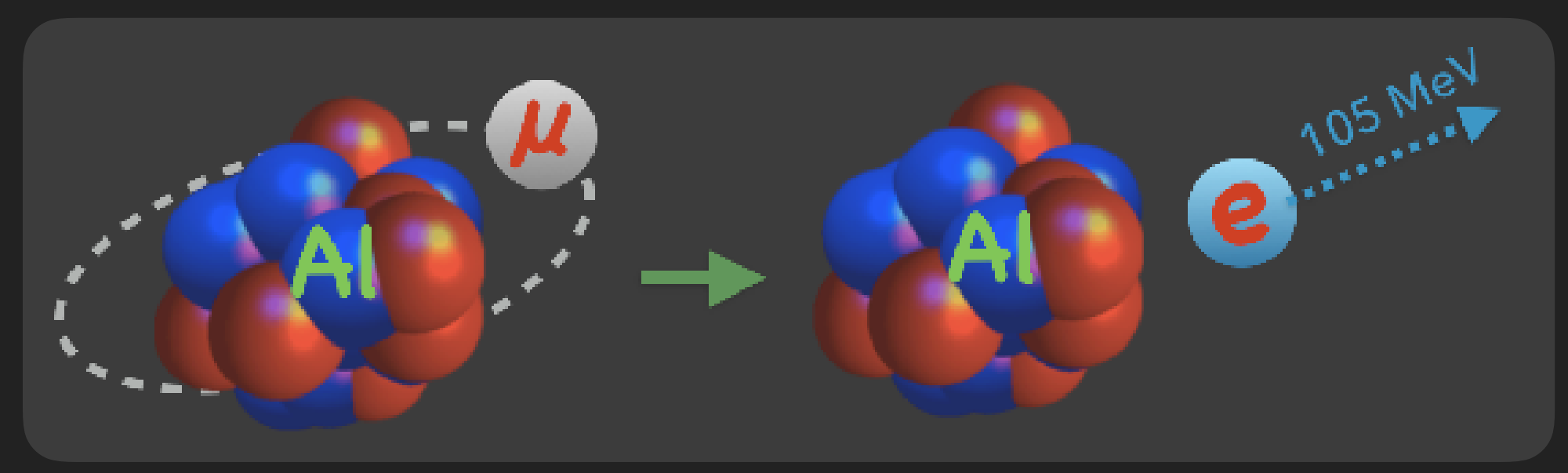




\section{What else will muonic Al do?}

\section{Nuclear Capture 61\%}

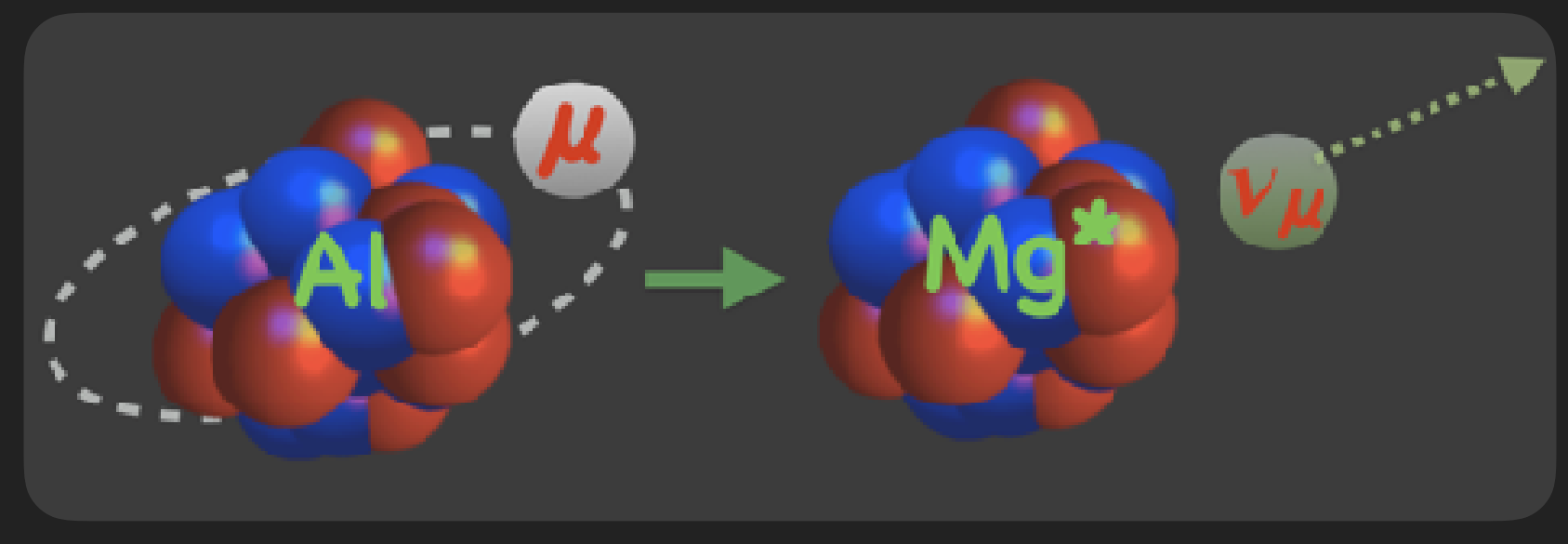

\section{Decay In Orbit (DIO) 39\%}

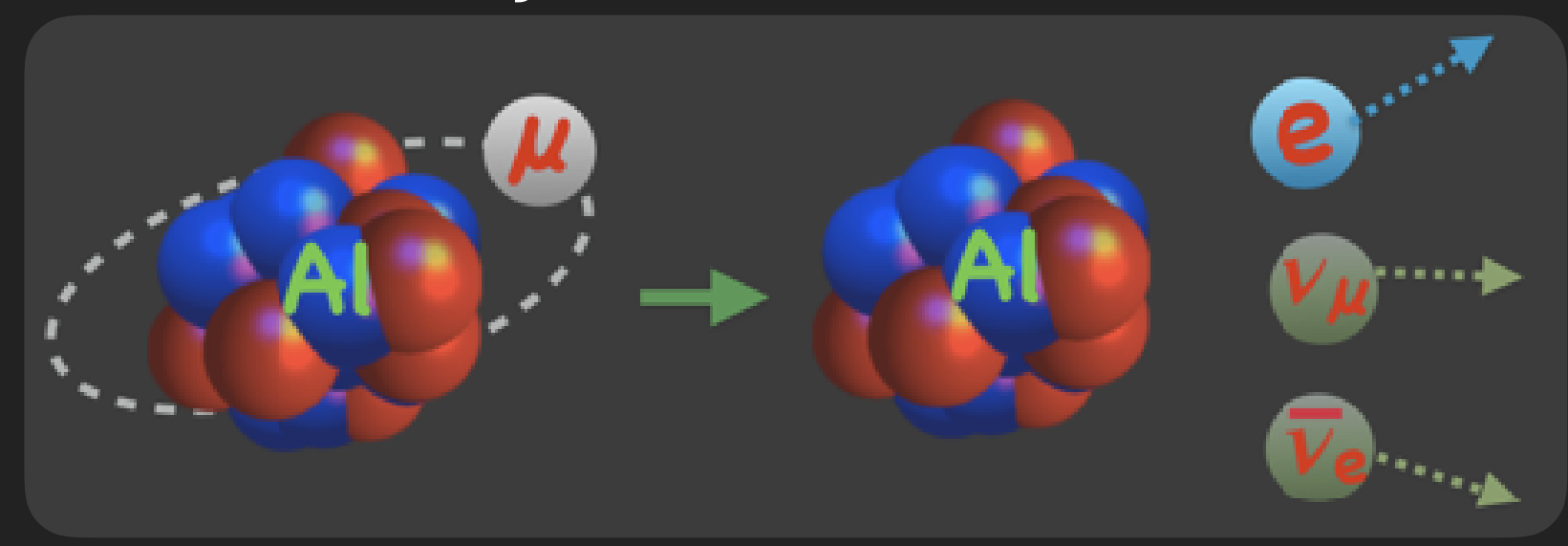

Conversion $<10-12$

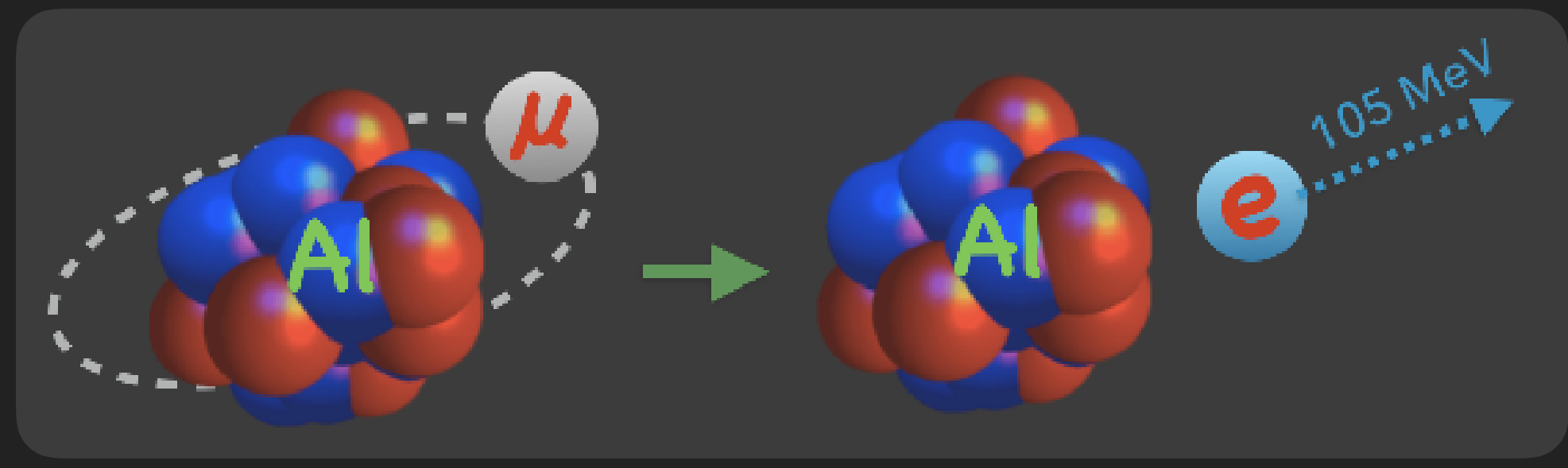




\section{What else will muonic Al do?}

Nuclear Capture 61\%

Normalization Factor

\section{Decay In Orbit (D|O) 39\%}

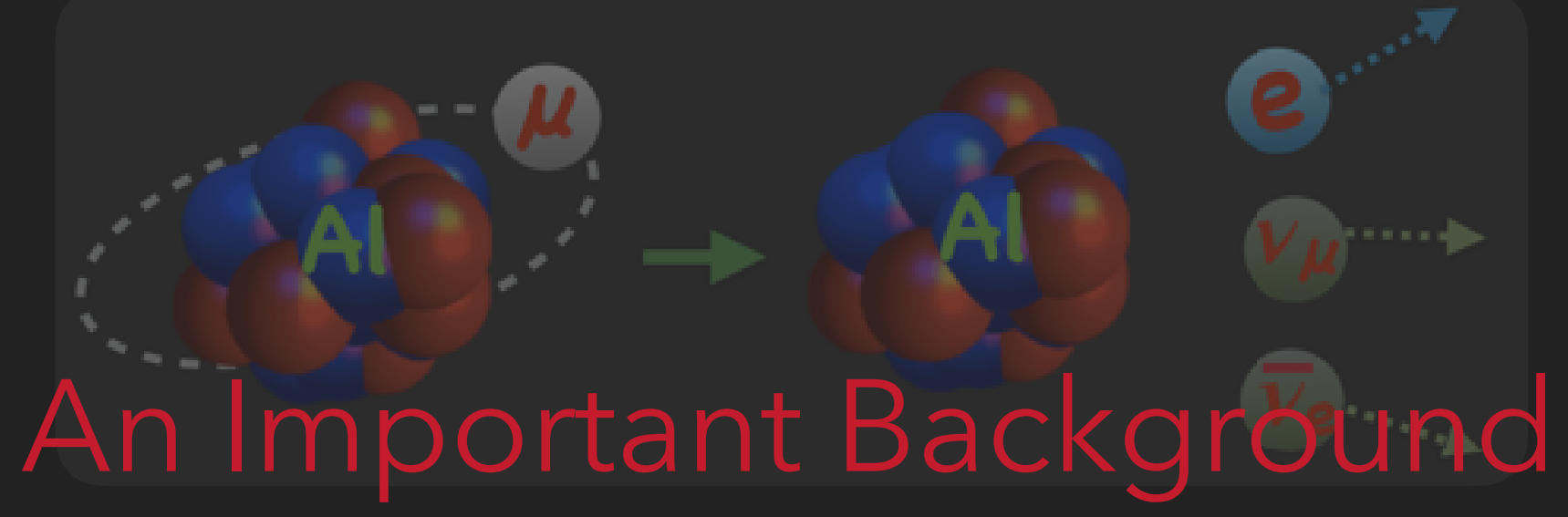

Conversion $<10^{-12}$

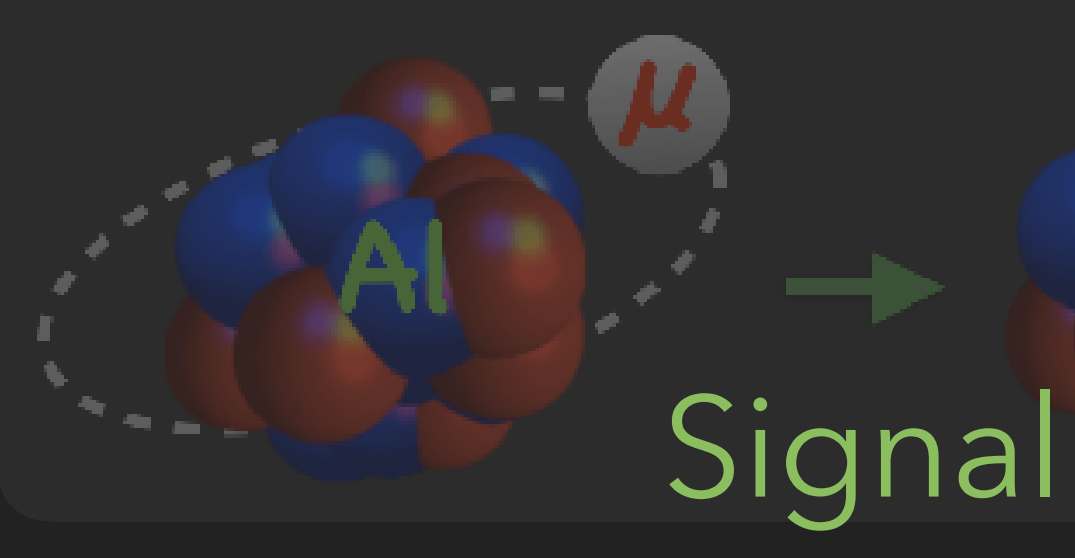




\section{Sensitivity Summary}

○ Previous experiments rule out $R_{\mu \mathrm{e}} \geqq 7 \times 10^{-13} @ 90 \% \mathrm{CL}$

○ Many NP models predict $R_{\mu \mathrm{e}} \sim 10^{-14}-10^{-16}$

○ If $R_{\mu e} \sim 10^{-15}$, we'll will see $\sim 40$ events

○ If $R_{\mu \mathrm{e}} \sim 3 \times 10^{-17}$, we should see 1 event (Single Event Sensitivity)

o Expected background is 0.4 an event

o Discovery reach $(5 \sigma): R_{\mu e} \geqq 2 \times 10^{-16}$

○ Exclusion power $(90 \% \mathrm{CL}): \mathrm{R}_{\mu \mathrm{e}} \geqq 8 \times 10^{-17}$ 
( ) Produce $10^{18}$ muonic ${ }^{27} \mathrm{Al}$ atoms

- Irradiate Al target with low energy $\mu$

- Only reconstruct $\sim 100 \mathrm{~K} \mu$ decays
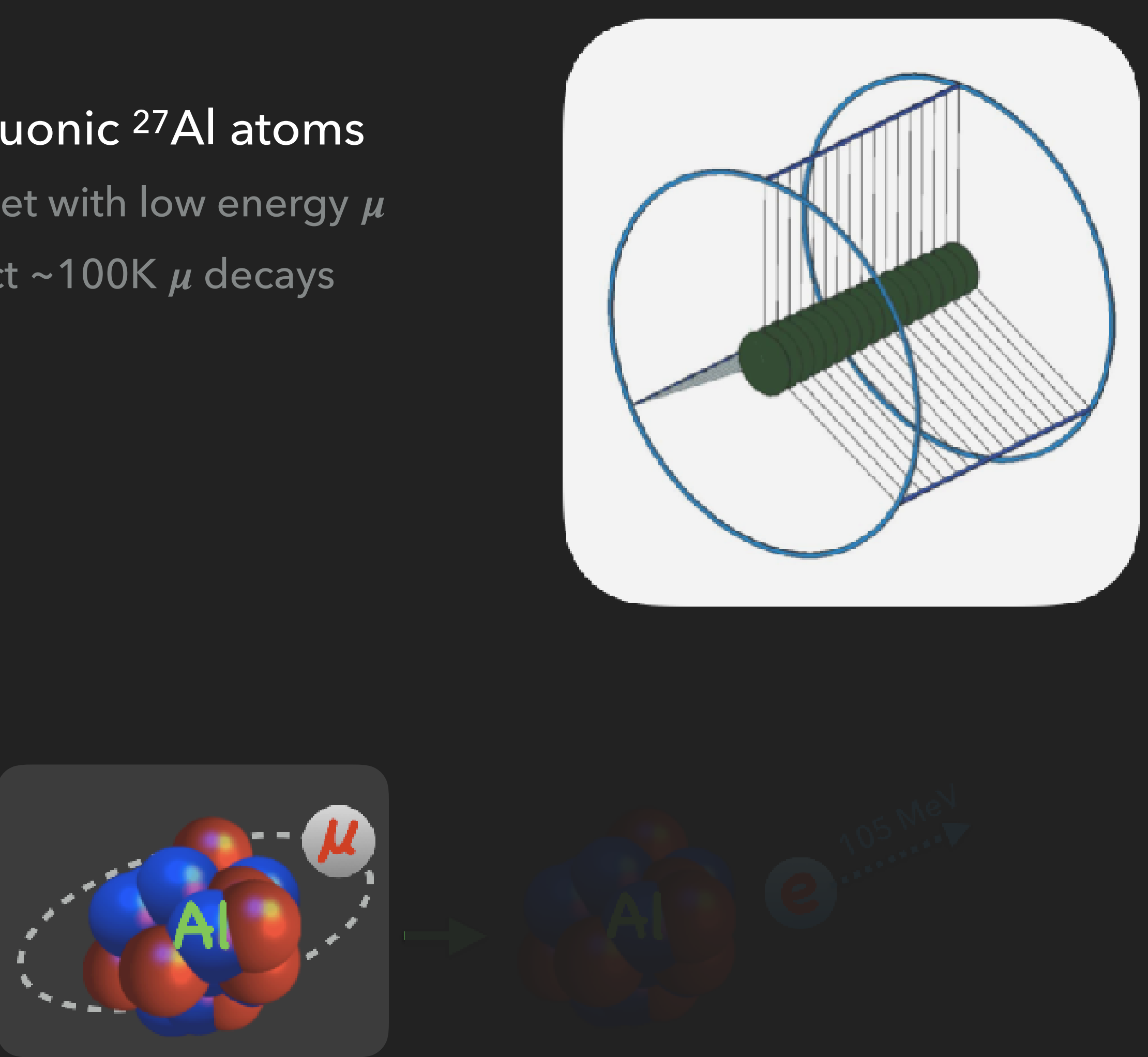


\section{The Basic Idea}

( ) Produce 1018 muonic ${ }^{27} \mathrm{Al}$ atoms

v Irradiate Al target with low energy $\mu$

- Only reconstruct $\sim 100 \mathrm{~K} \mu$ decays

o Count conversion-like electrons

v Tracking and calorimetry
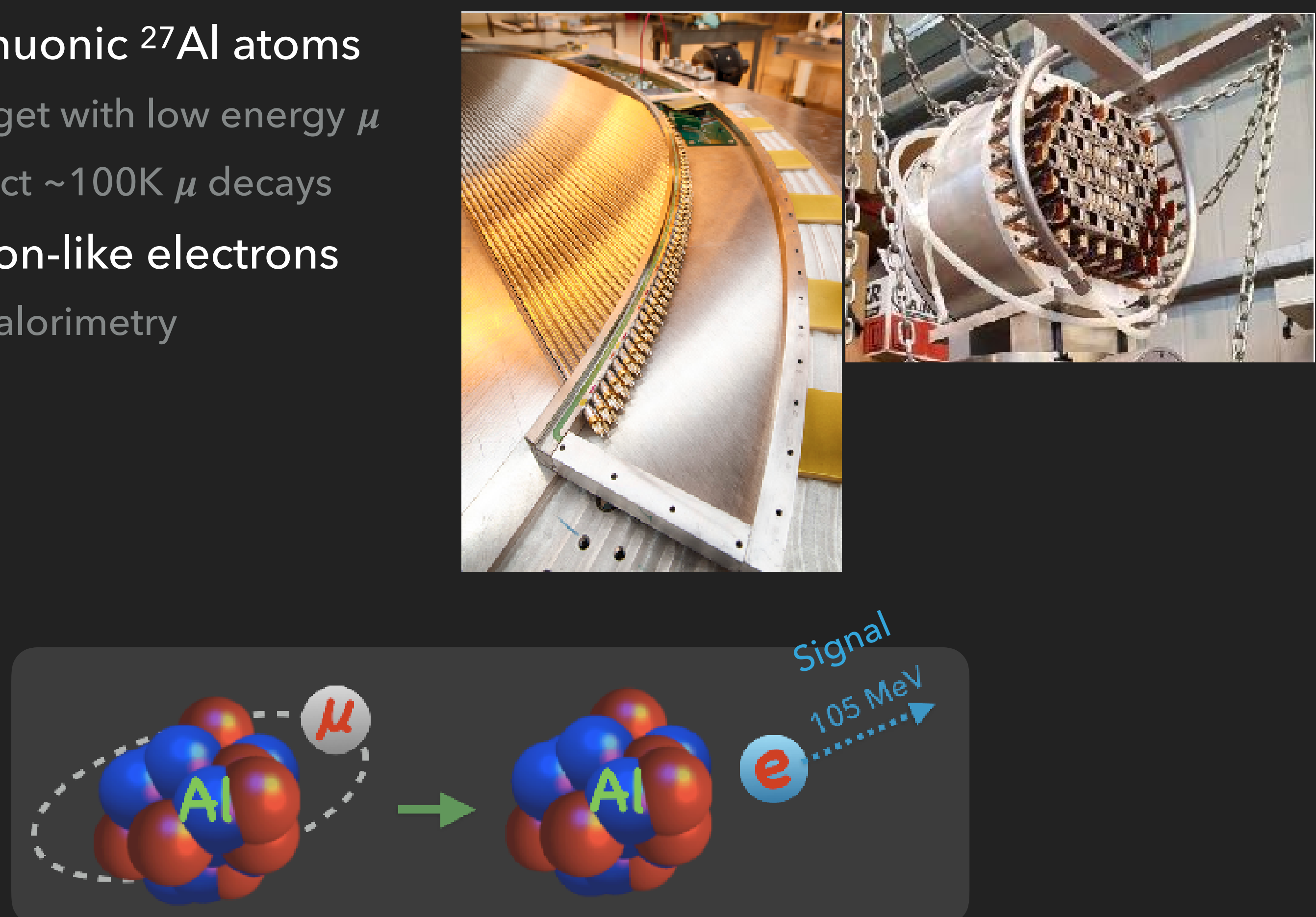


\section{The Basic Idea}

( ) Produce $10^{18}$ muonic ${ }^{27} \mathrm{Al}$ atoms

- Irradiate Al target with low energy $\mu$

- Only reconstruct $\sim 100 \mathrm{~K} \mu$ decays

o Count conversion-like electrons - Tracking and calorimetry

o Suppress experimental backgrounds
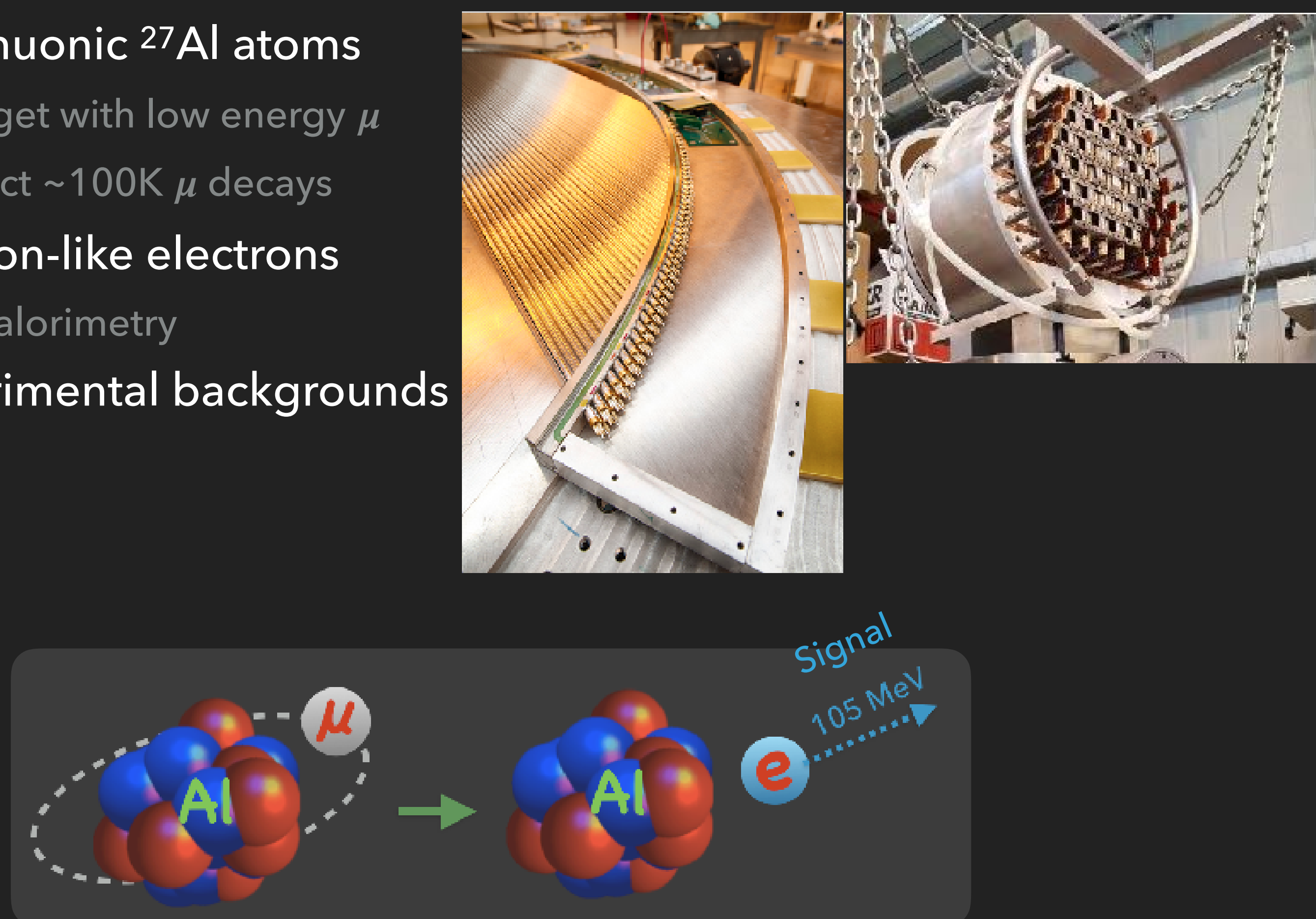


\section{A Glance at the Experiment}
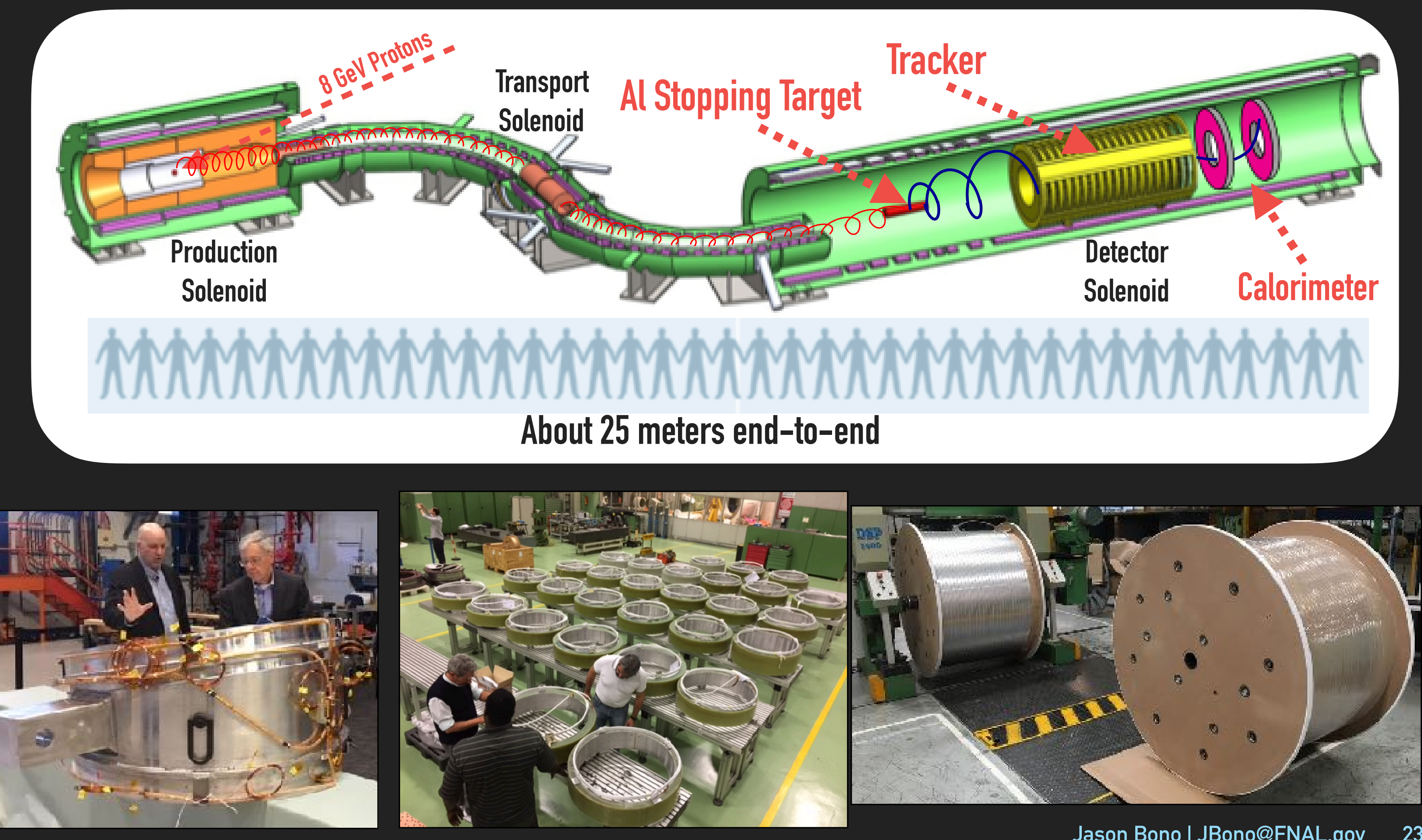


\section{A Glance at the Experiment}

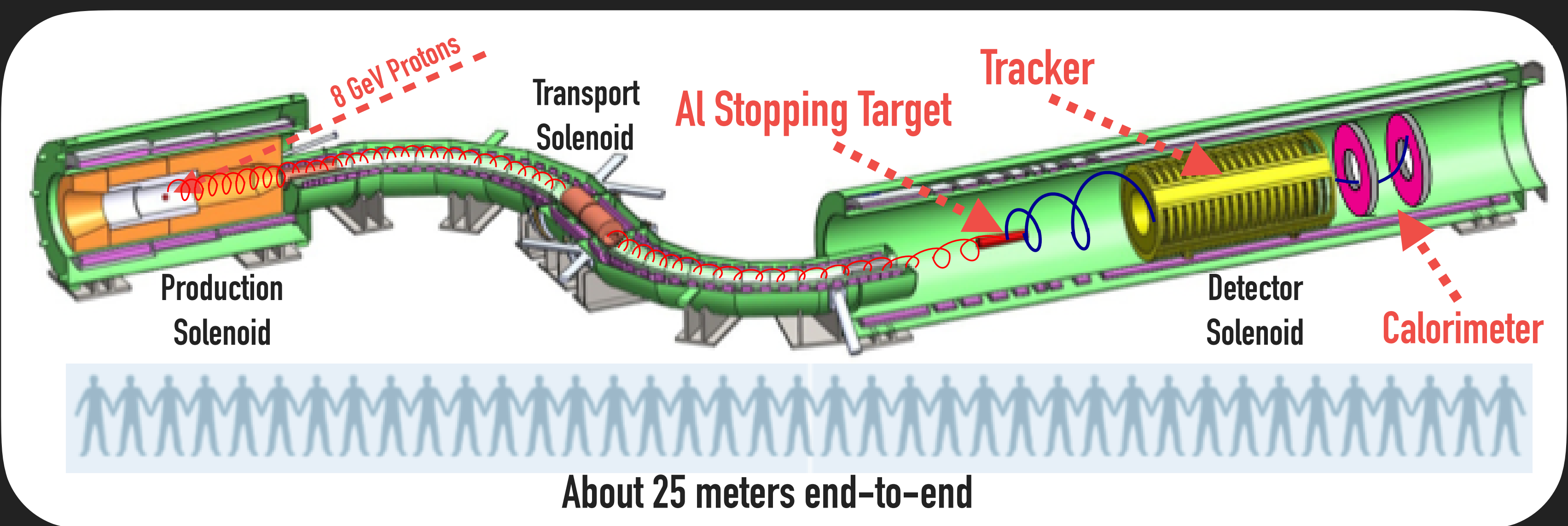

( ) First issue: scaling from previous experiments, to get $10^{18}$ muons with an $8 \mathrm{~kW}$ proton beam, it seems that we'd have to run for thousands of years

( This brings us to our first big idea 


\section{First Big Idea — The World's Hottest Muon Source}

○ $8 \mathrm{GeV}, 8 \mathrm{~kW}$ proton beam Incident on tungsten target

O Confine the soft pions with a solenoidal B-field

○ $\operatorname{BR}\left(\pi^{-} \rightarrow \boldsymbol{\mu}^{-} \boldsymbol{\nu}_{\mu}\right) \sim 99.9 \%$

o Include a strong gradient to increase the yield further through magnetic reflection

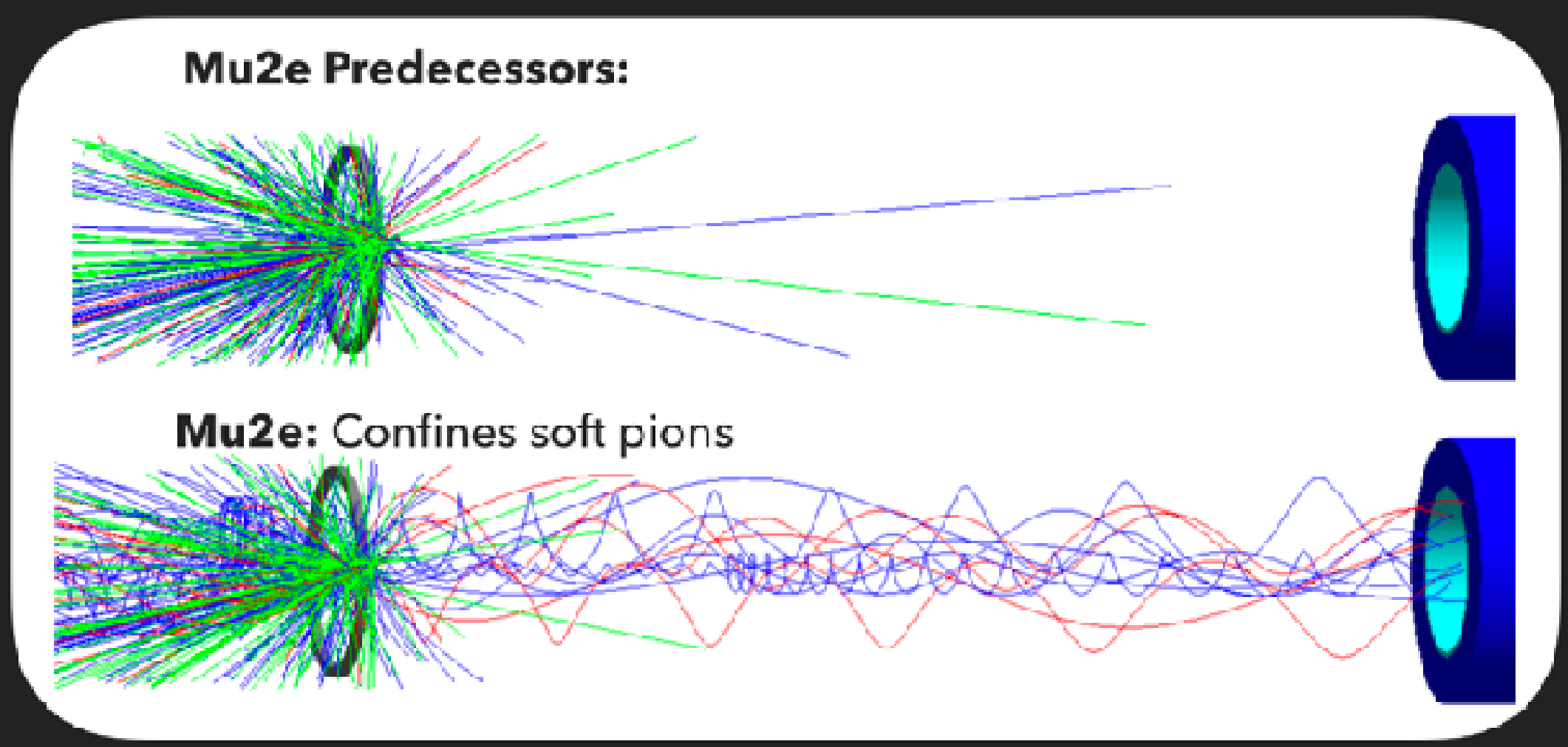

Concept originated by R.M. Dzhilkibaev, V.M. Lobashev, Sov.J.Nucl.Phys 49, 384 (1989) 


\section{Enter the Detector Solenoid}

o Next destination for the beam: the Al stopping target!

o $10^{20}$ POT in 3 years $\rightarrow 10^{18}$ stopped $\mu$, as required

> $10^{10}$ stopped $\mu / \mathrm{s} !$
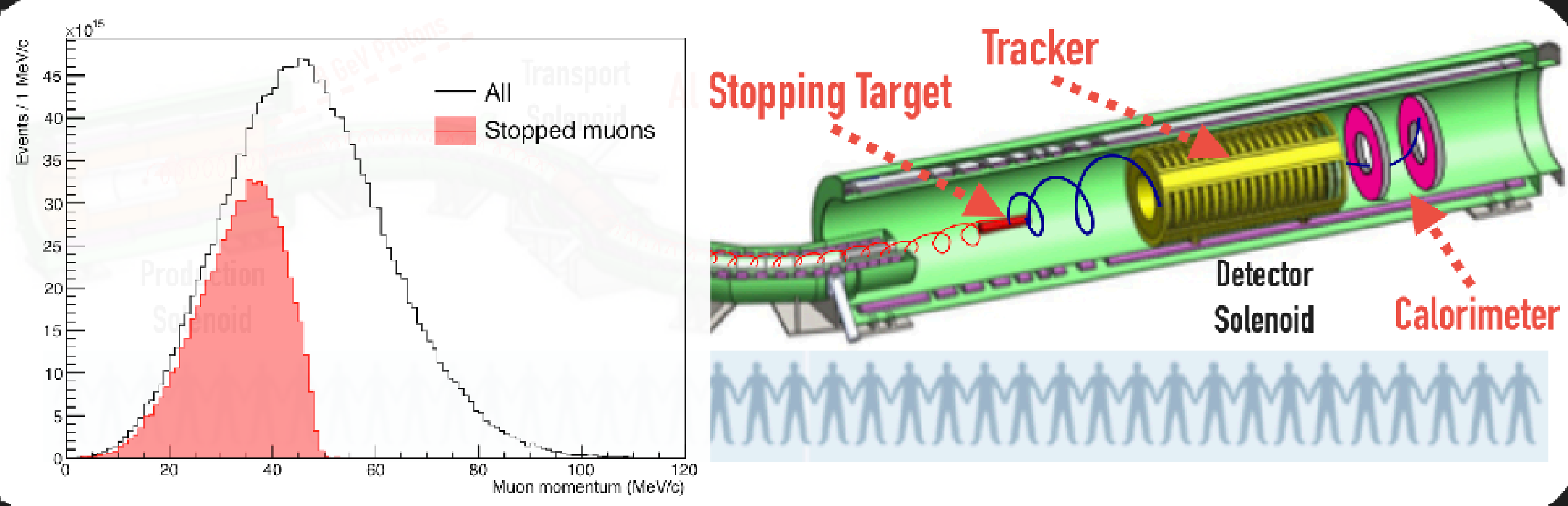


\section{Prompt Background: Radiative Pion Capture}

o The muon beam is naturally contaminated with its parent pions

o Some pions are stopped on the Al target, or other parts of the detector

( ) $\sim 2 \%$ of these produce a photon or an e-e pair, and sometimes the e will look like a CE!

(- But, the pion lifetime is around 26 ns, while the muon lifetime in Al is 864 ns

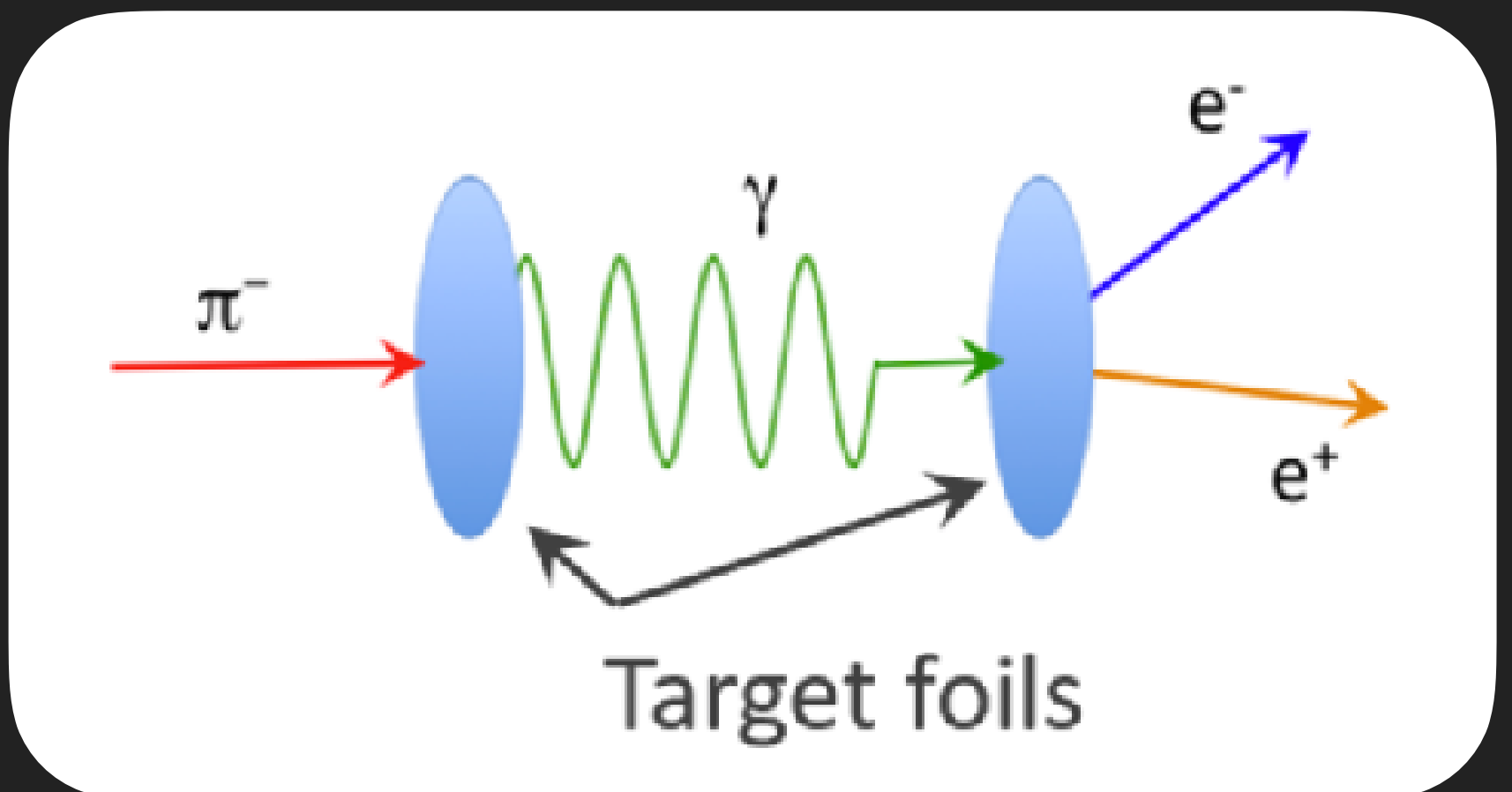

This leads us to our second "big idea" 


\section{Second Big Idea — A Pulsed Proton Beam with Extinction}

O Use a pulsed beam with a long interval (1.7 $\mu \mathrm{s})$ between bunches

o Delay the search window

- Muonic aluminum ( $\tau \sim 0.864 \mu \mathrm{s}$ ) is well optimized for the beam structure!

- $39 \mathrm{M}$ protons / pulse

(.) Extinction: out-of-time protons / in-time protons $=10^{-11}$

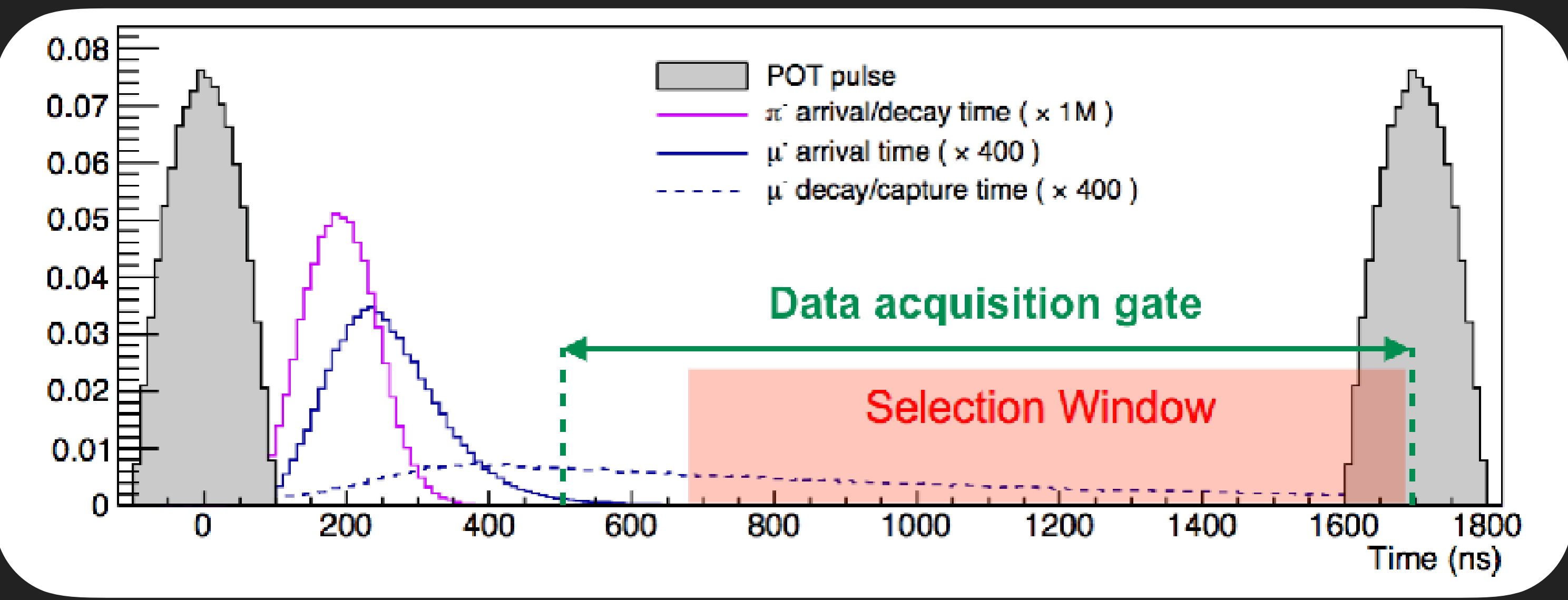




\section{Cosmic Background}

- $10^{9}$ cosmic rays / day

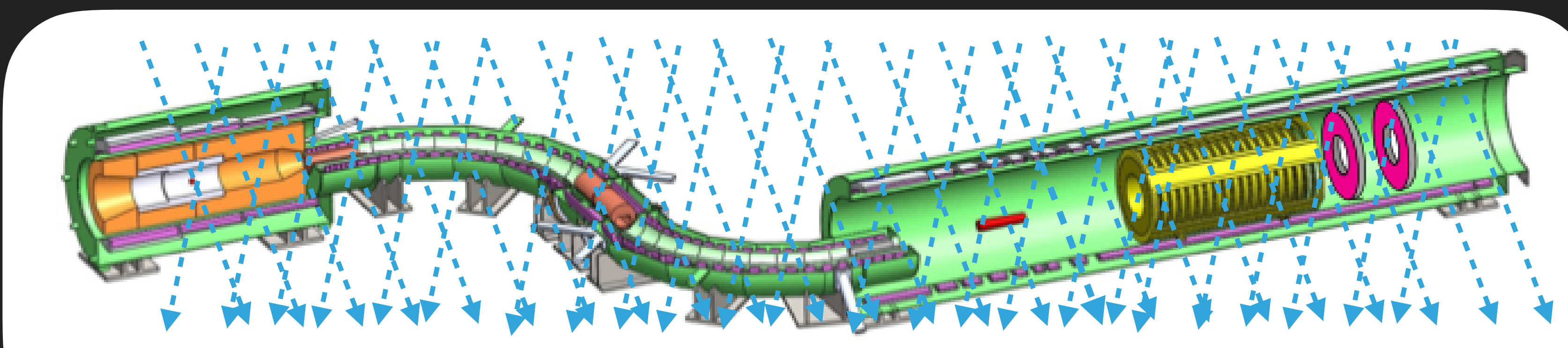




\section{Cosmic Background}

O $10^{9}$ cosmic rays / day

- Fiducial cuts help, but we still get 1 BG event/day

- Experiment killer!

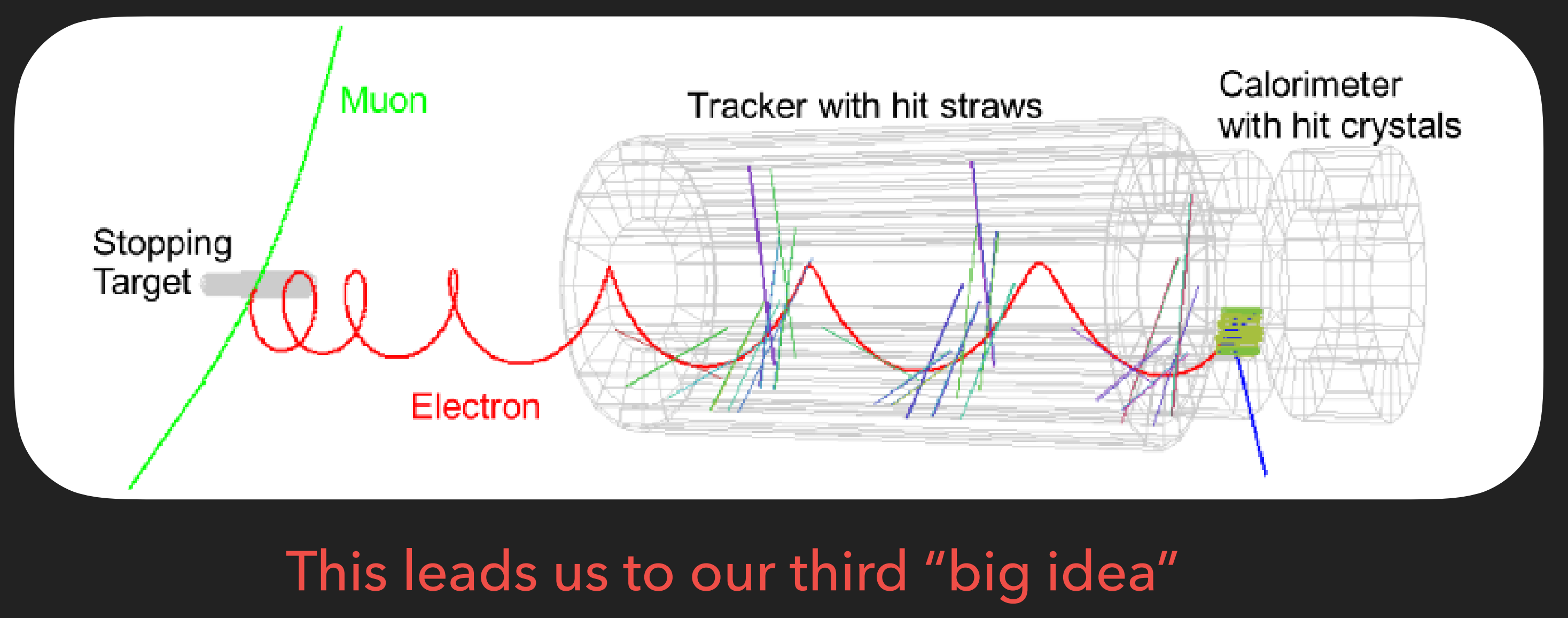




\section{Third Big Idea — An Efficient Cosmic Ray Veto System}

- 4 layers of extruded polystyrene scintillator counter with wavelength shifting fibers - readout $2 \mathrm{~mm} \times 2 \mathrm{~mm}$ SiPMs

- Coincidence $(15 \mathrm{~ns}, 30 \mathrm{~cm})$ in 3 of the 4 layers

o $99.99 \%$ efficiency requirement: better not have any holes!

- Currently the largest background in the experiment

o Minimize false veto

- Require $<10 \%$ dead-time
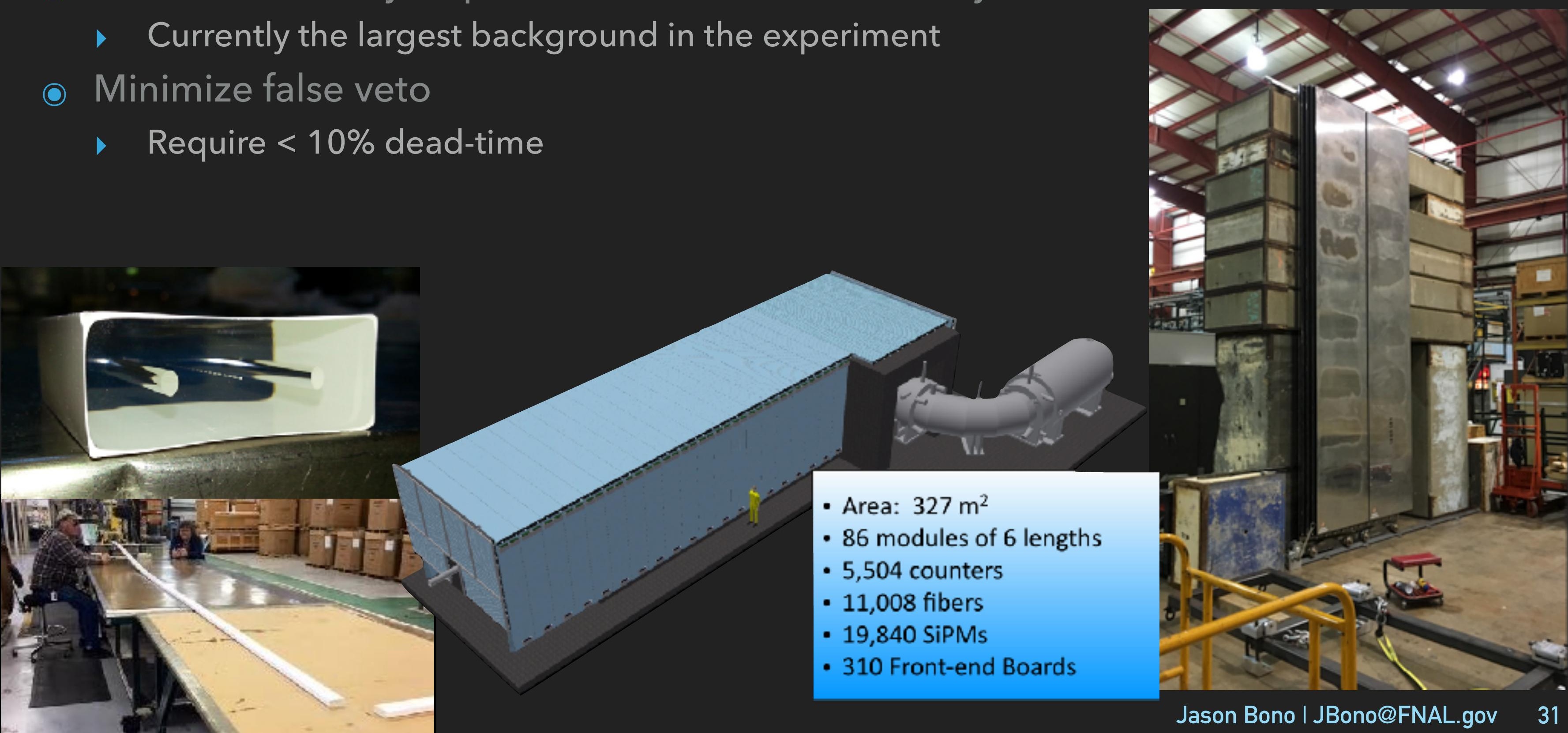


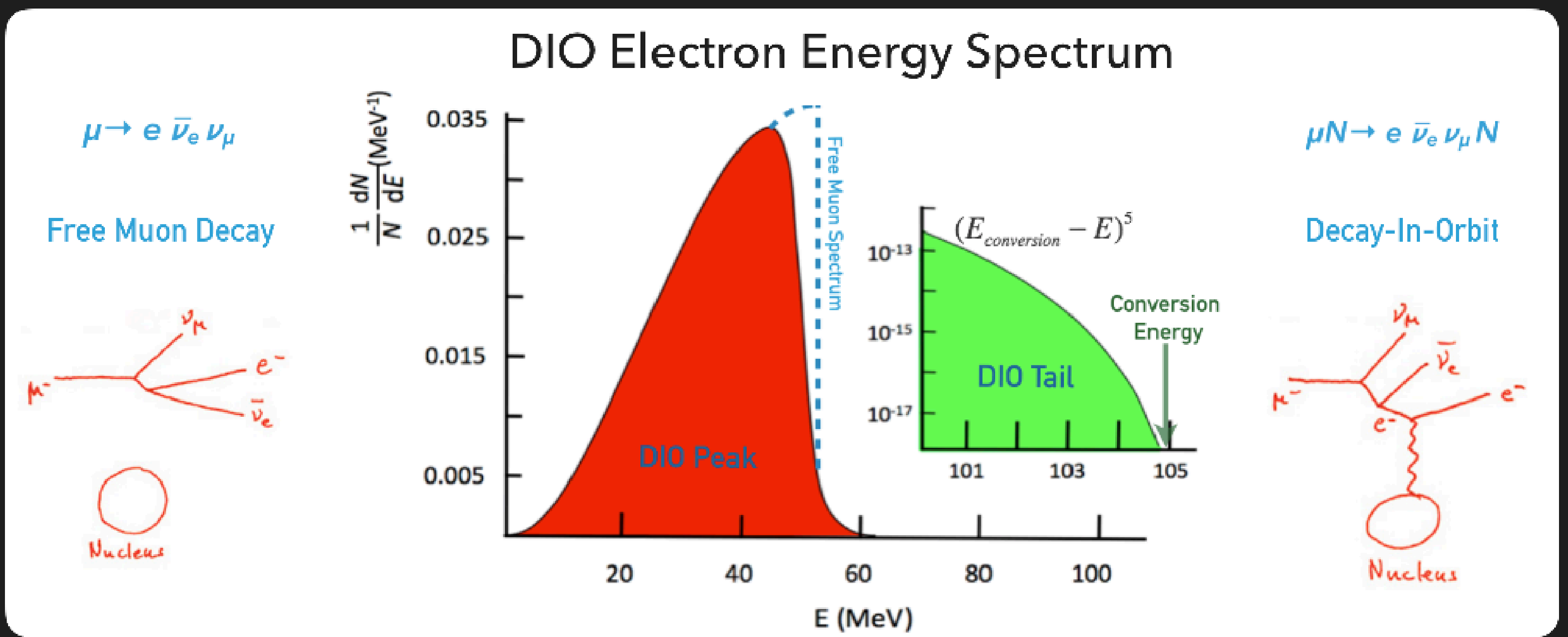

For a theory prediction, see: R.Szafron, A. Czarnecki, Phys. Rev. D94(2016)051301 


\section{Decay In Orbit Background}

o Other than momentum, there is no way to distinguish DIO from CE

- Fiducial cuts don't help

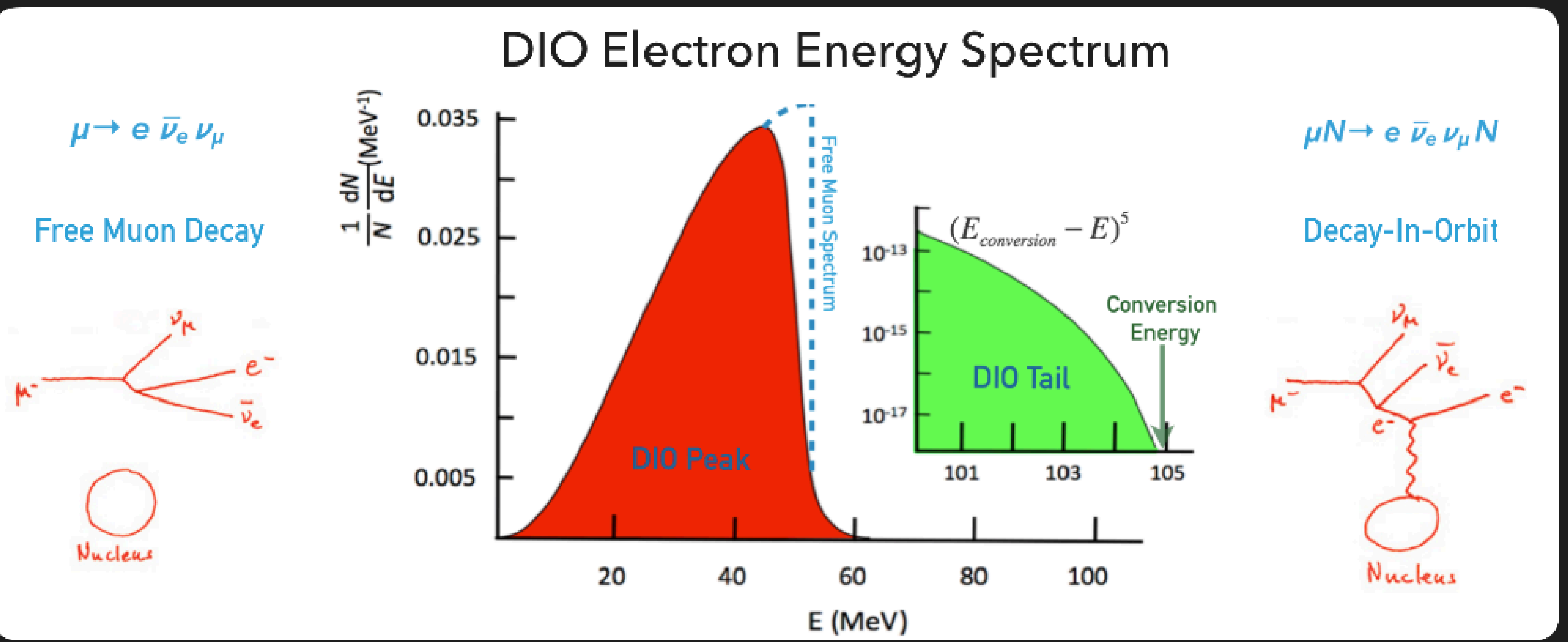

For a theory prediction, see: R.Szafron, A. Czarnecki, Phys. Rev. D94(2016)051301 


\section{Fourth Big Idea — Wrestling With DOls via Tracking}

- Be blind to, and protect from, low momentum electrons

- Have an annular geometry + operate in a $1 \mathrm{~T}$ field

• $\mathrm{d} p / d t=q(E+v \times B) \rightarrow R=p_{\perp} / q B$

- Require excellent resolution \& acceptance

> $\sigma<0.2 \%$ @ $105 \mathrm{MeV}$

- (Acceptance)x(Reco efficiency) $\sim 20 \%$

- Minimize scattering and energy loss

- Ultra low mass

- Operate in vacuum ( $10^{-4}$ torr $)$
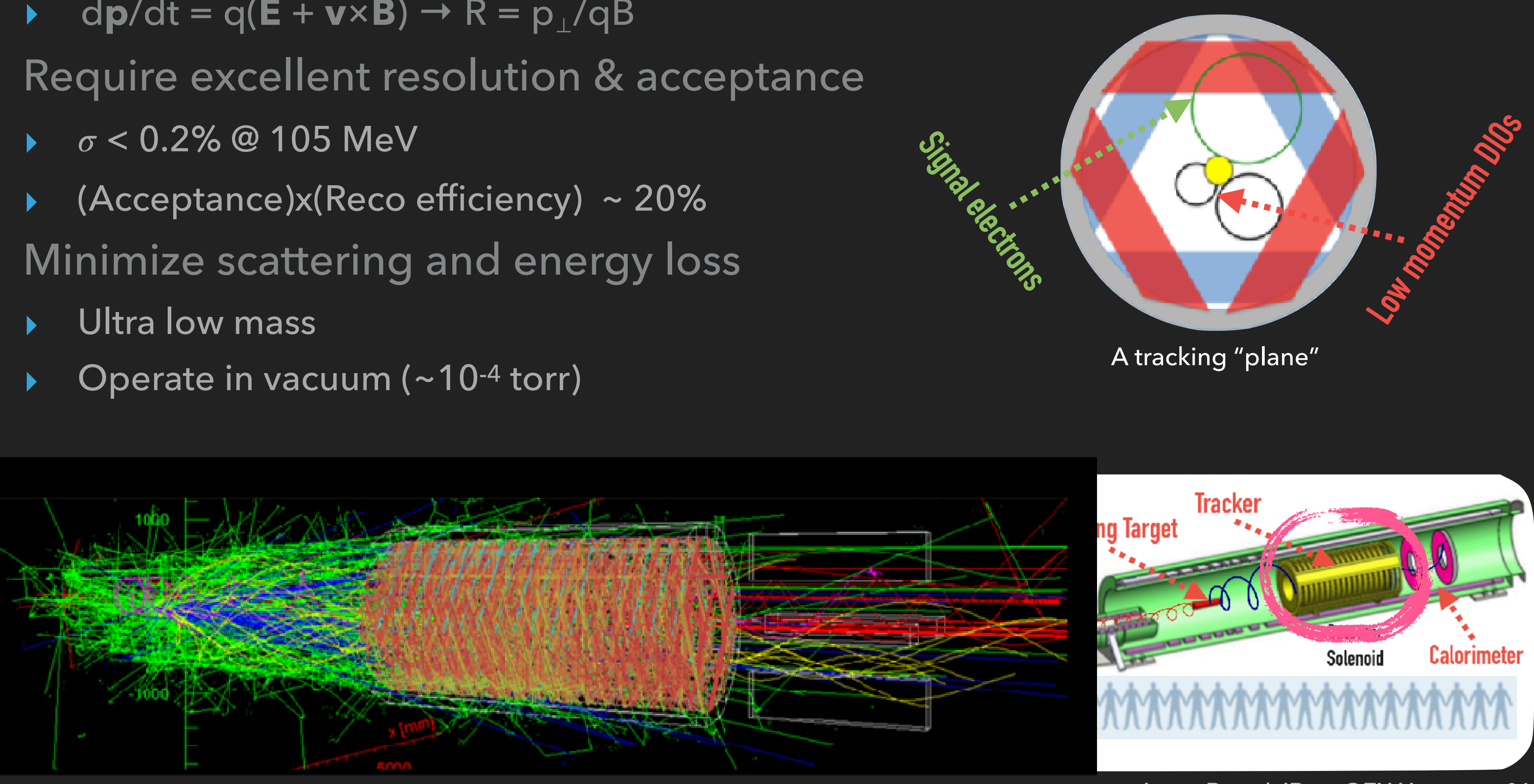


\section{$\sim 20,000$ straw-tubes running perpendicular the B-field}

- $5 \mathrm{~mm}$ diameter, metalized mylar straws

\ $15 \mu \mathrm{m}$ wall thickness

1 $\sim 1.2 \mathrm{~m}$ to $\sim 0.5 \mathrm{~m}$ in length

- Modular design

- Tracking region: $38<\mathrm{r}<70 \mathrm{~cm}$

0 Will be the largest, lowest density, straw-tube detector ever to be held in vacuum

v The drift gas alone is $\sim 7 \%$ of the mass

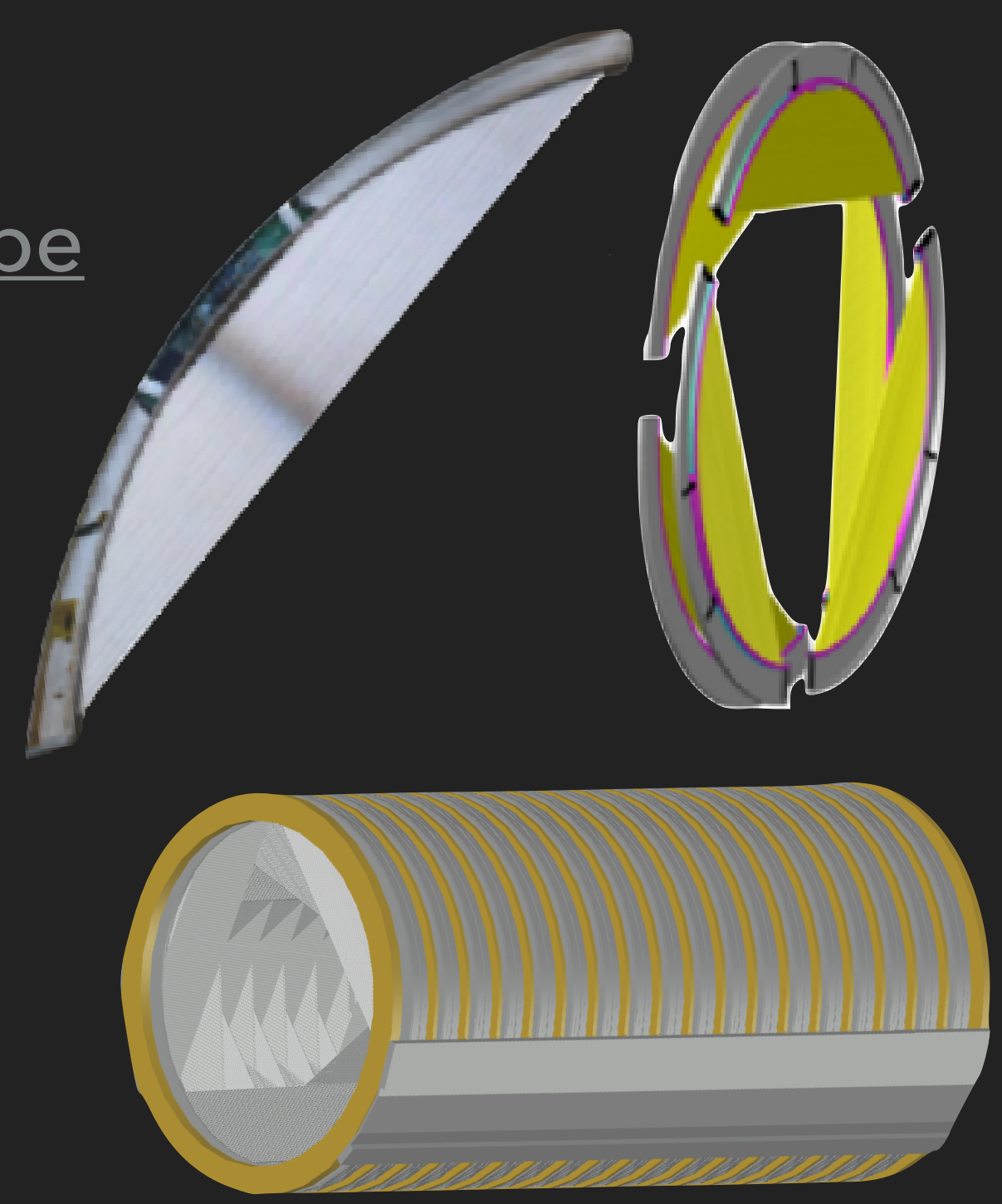




\section{8-Straw}

Prototype
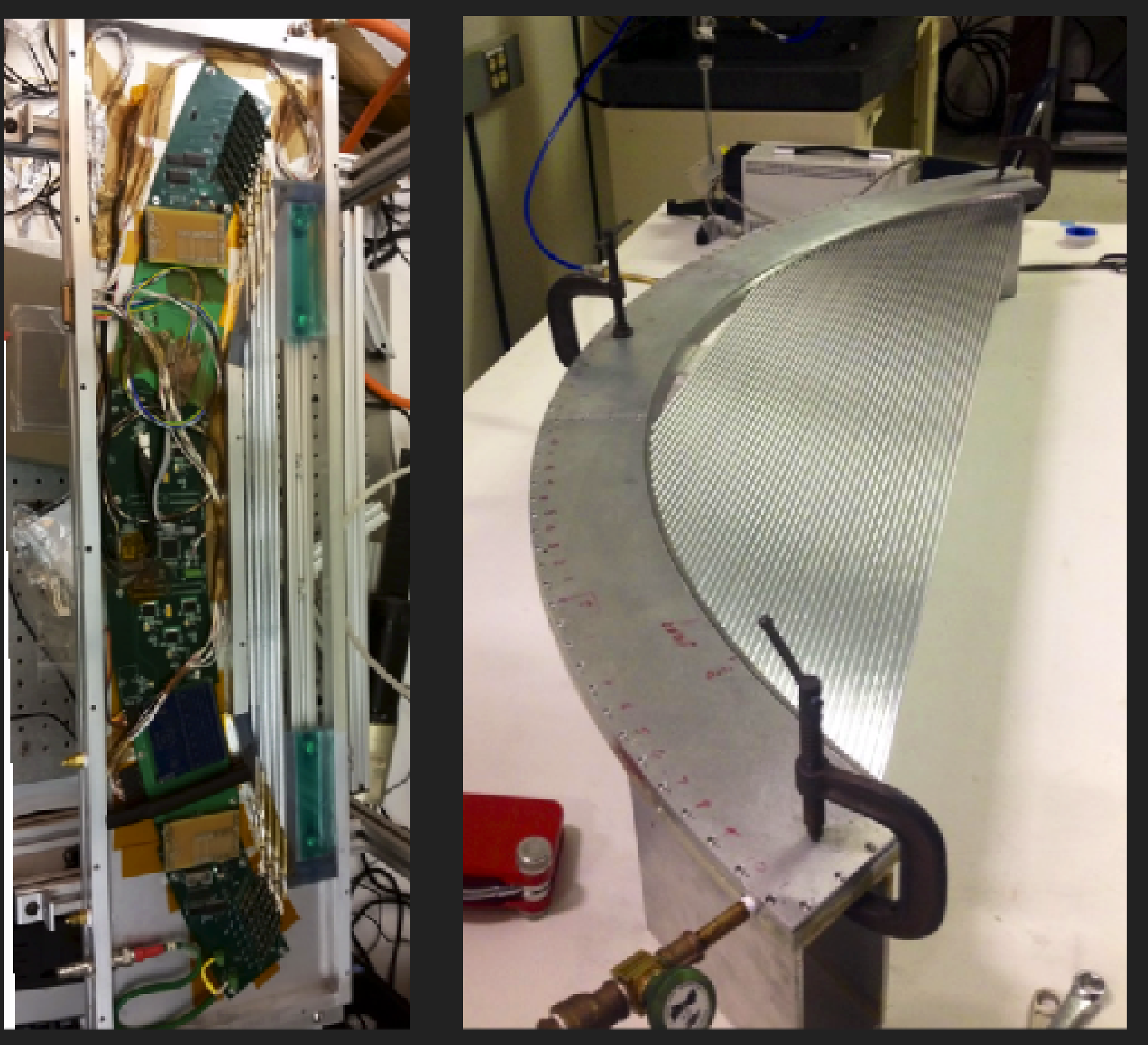

Rectangular Prototype 2

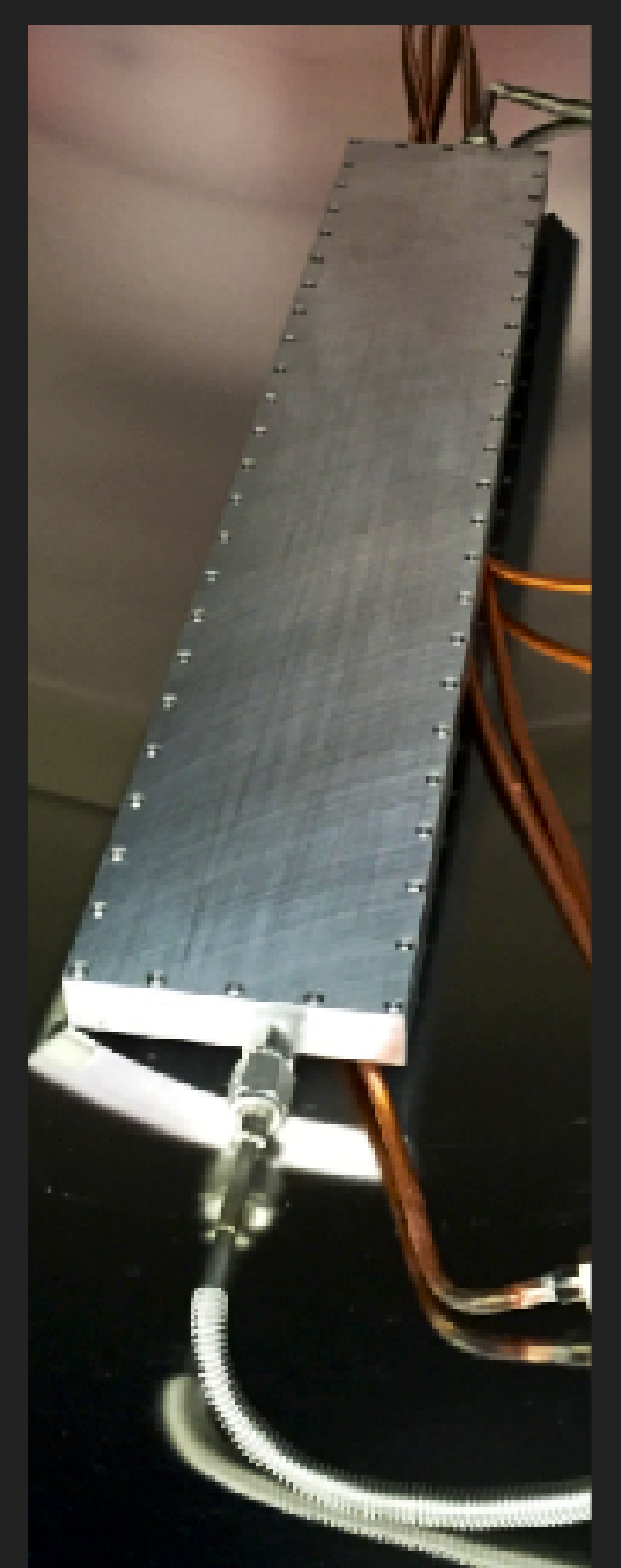

Full Panel V2.5

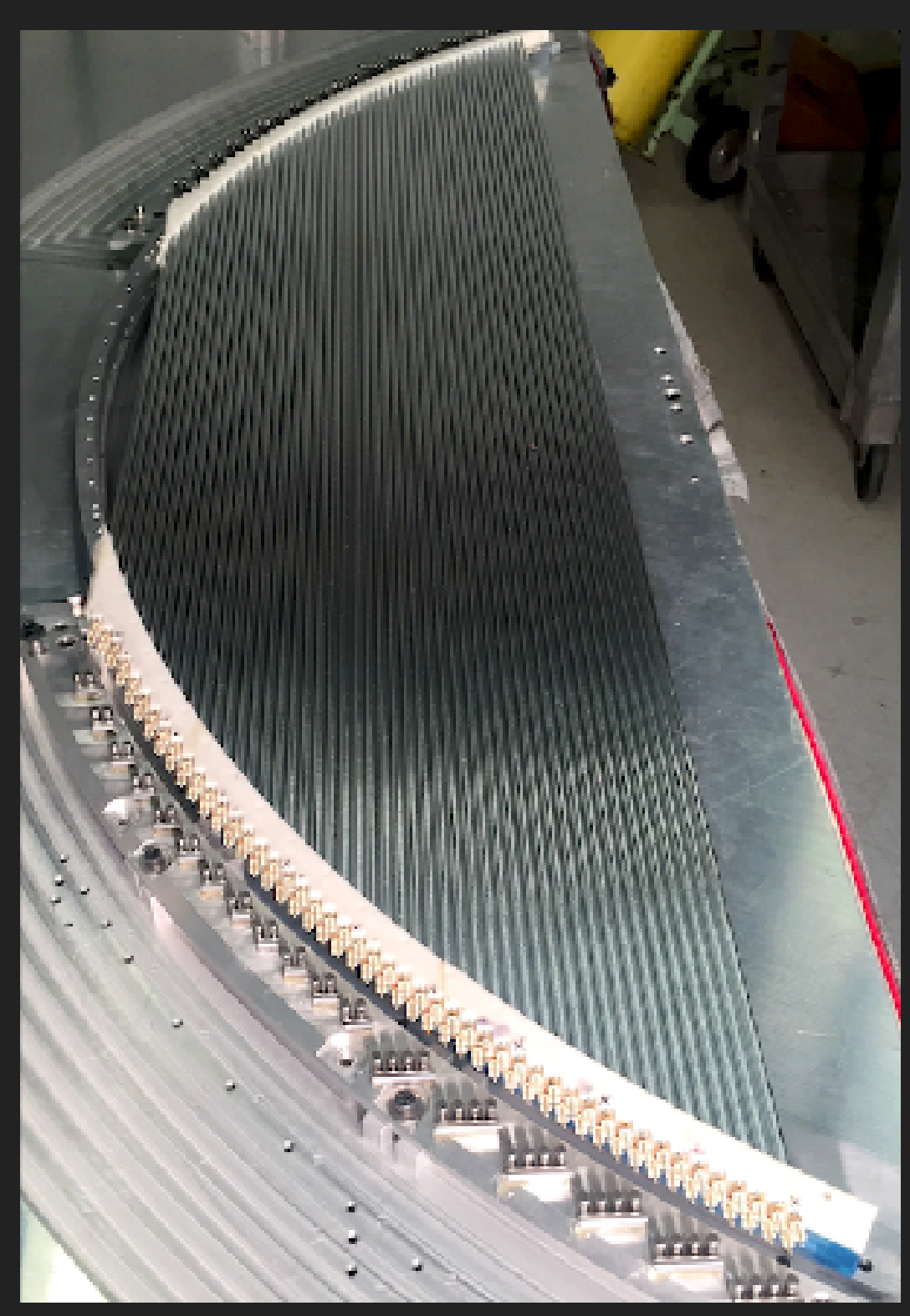

Early 2015

Completion Date

Mid 2017 


\section{Module production has begun}

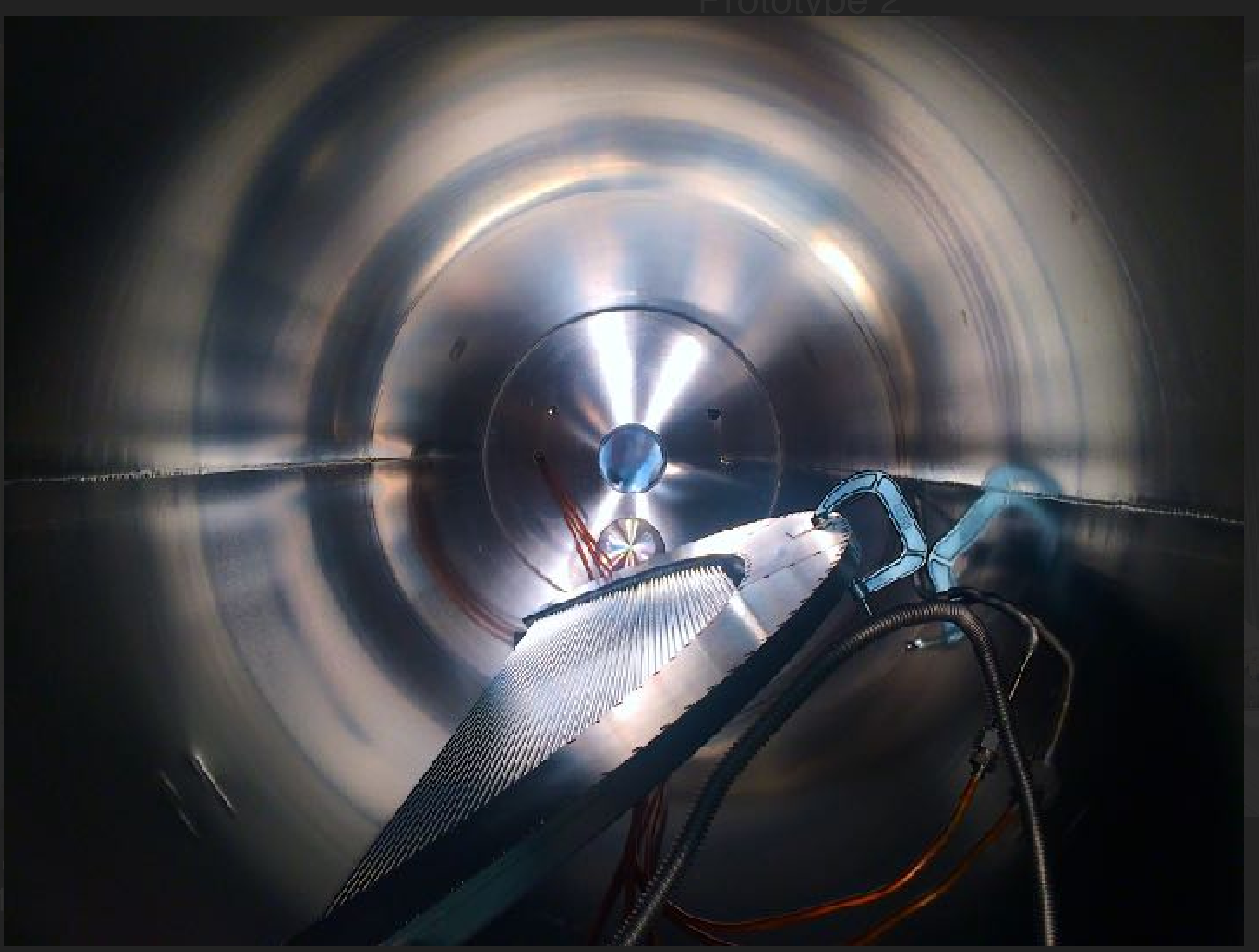




\section{(0) Discovery reach $(5 \sigma): \mathrm{R}_{\mu \mathrm{e}} \gtrsim 2 \times 10-16$ \\ ( ) Exclusion power $(90 \% \mathrm{CL}): \mathrm{R}_{\mu \mathrm{e}} \approx 8 \times 10^{-17}$}

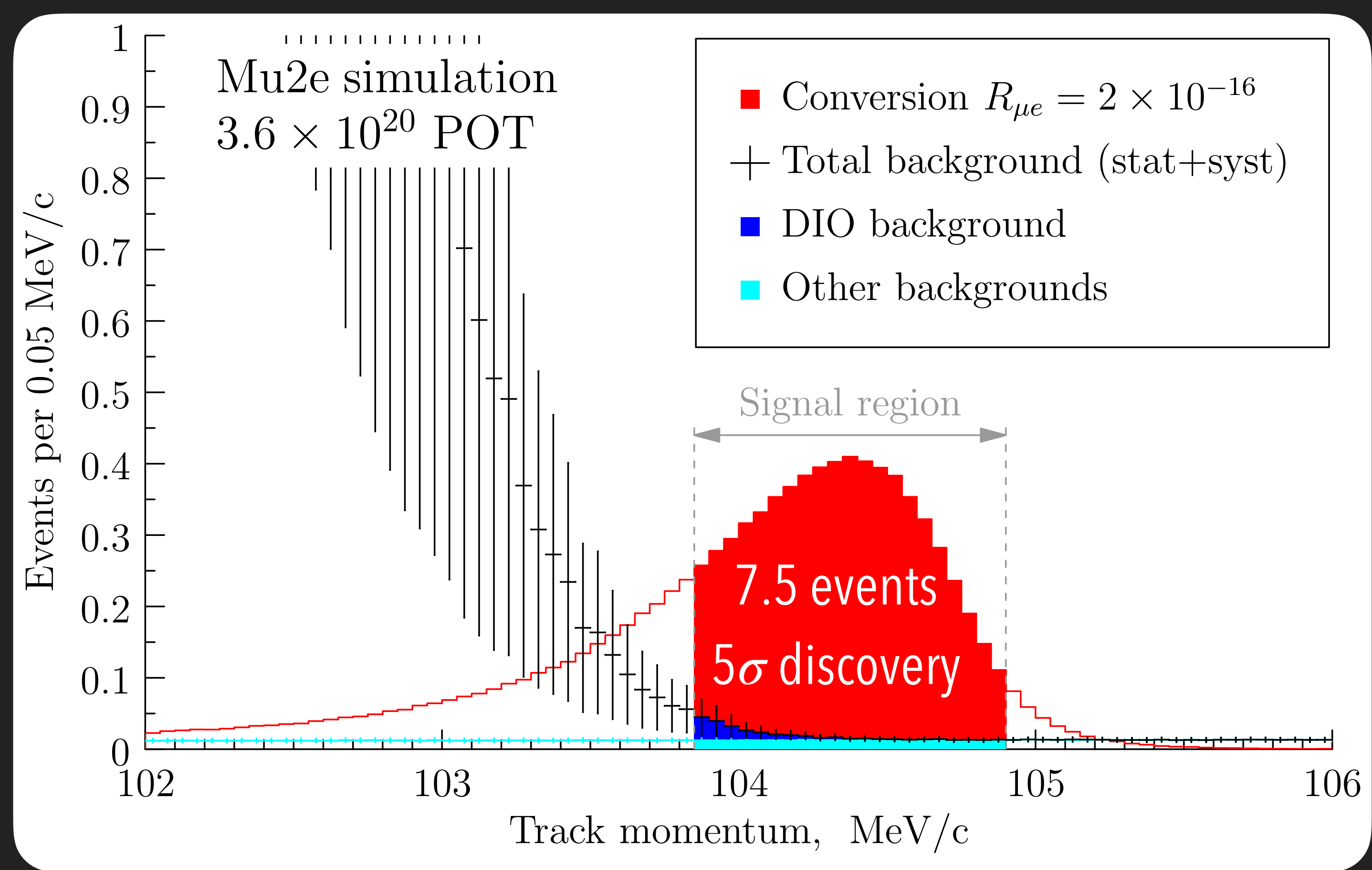




\section{Outline}

2

\section{Mu2e}
o ELFV
O Experimentat Betails
○ The Future 


\section{What if Mu2e sees a signal?}
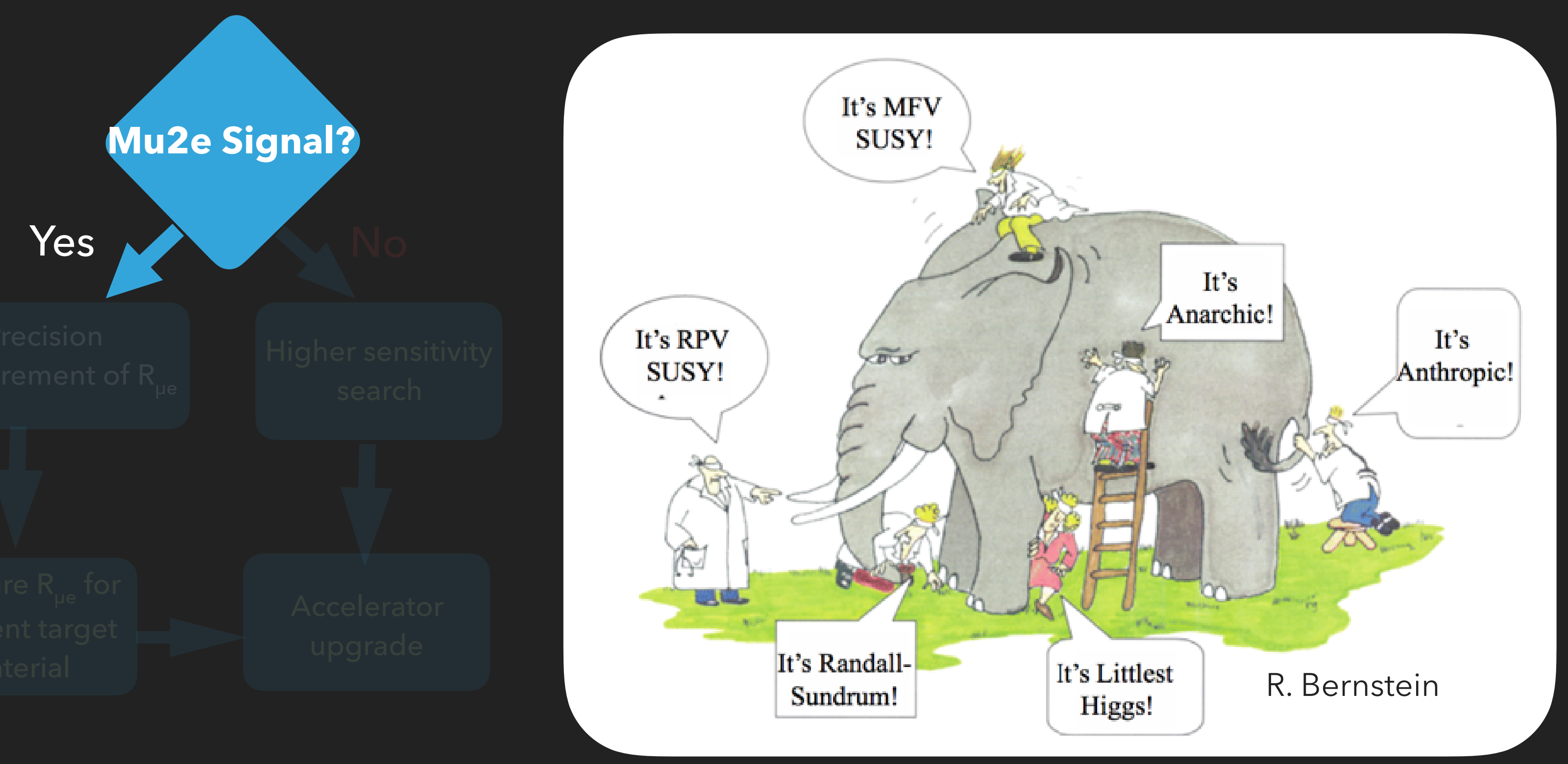


\section{$\mathrm{R}_{\mu \mathrm{e}}$ in multiple nuclei is a powerful model discriminator}

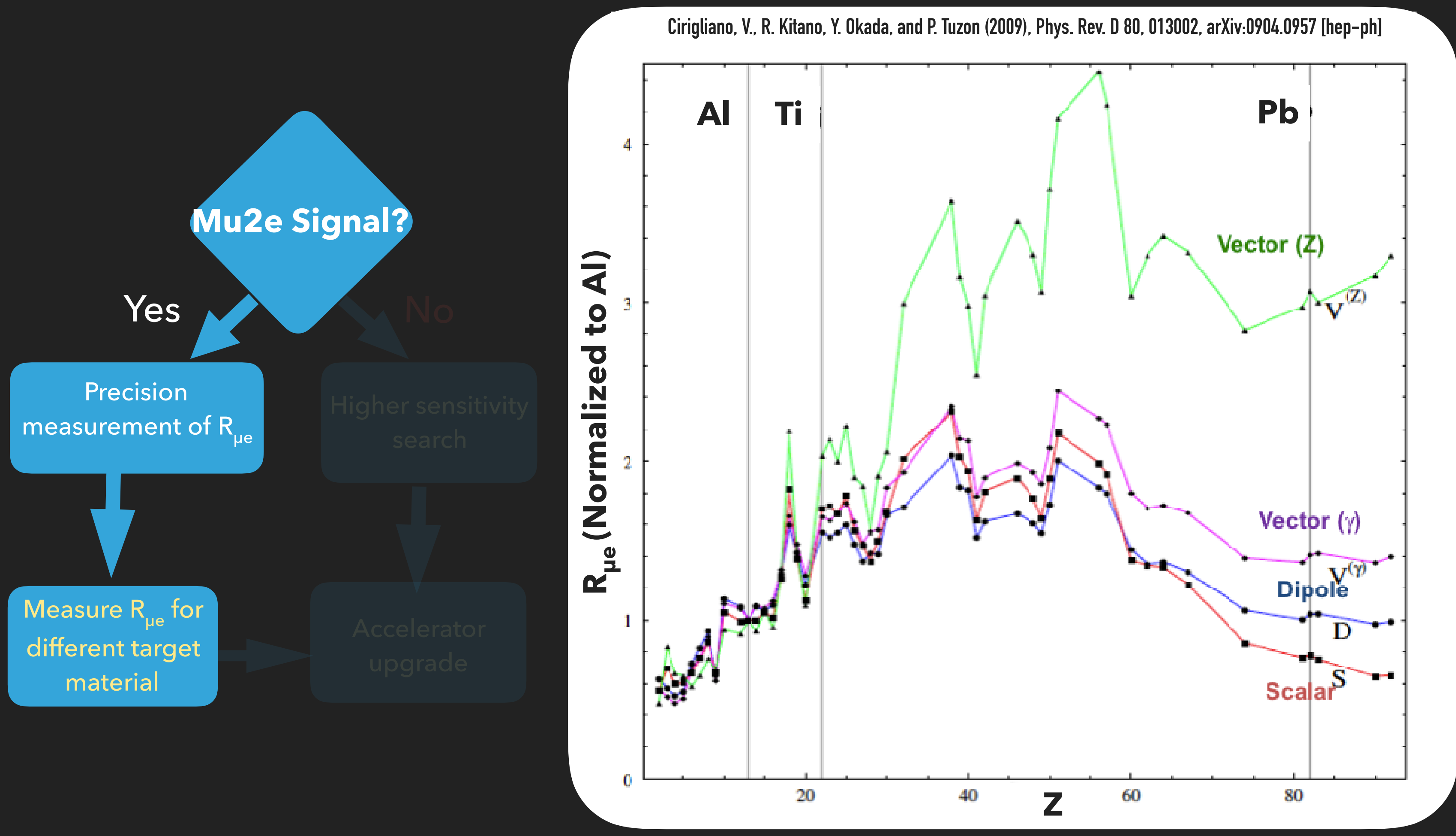




\section{A Mu2e upgrade is well motivated in any scenario}

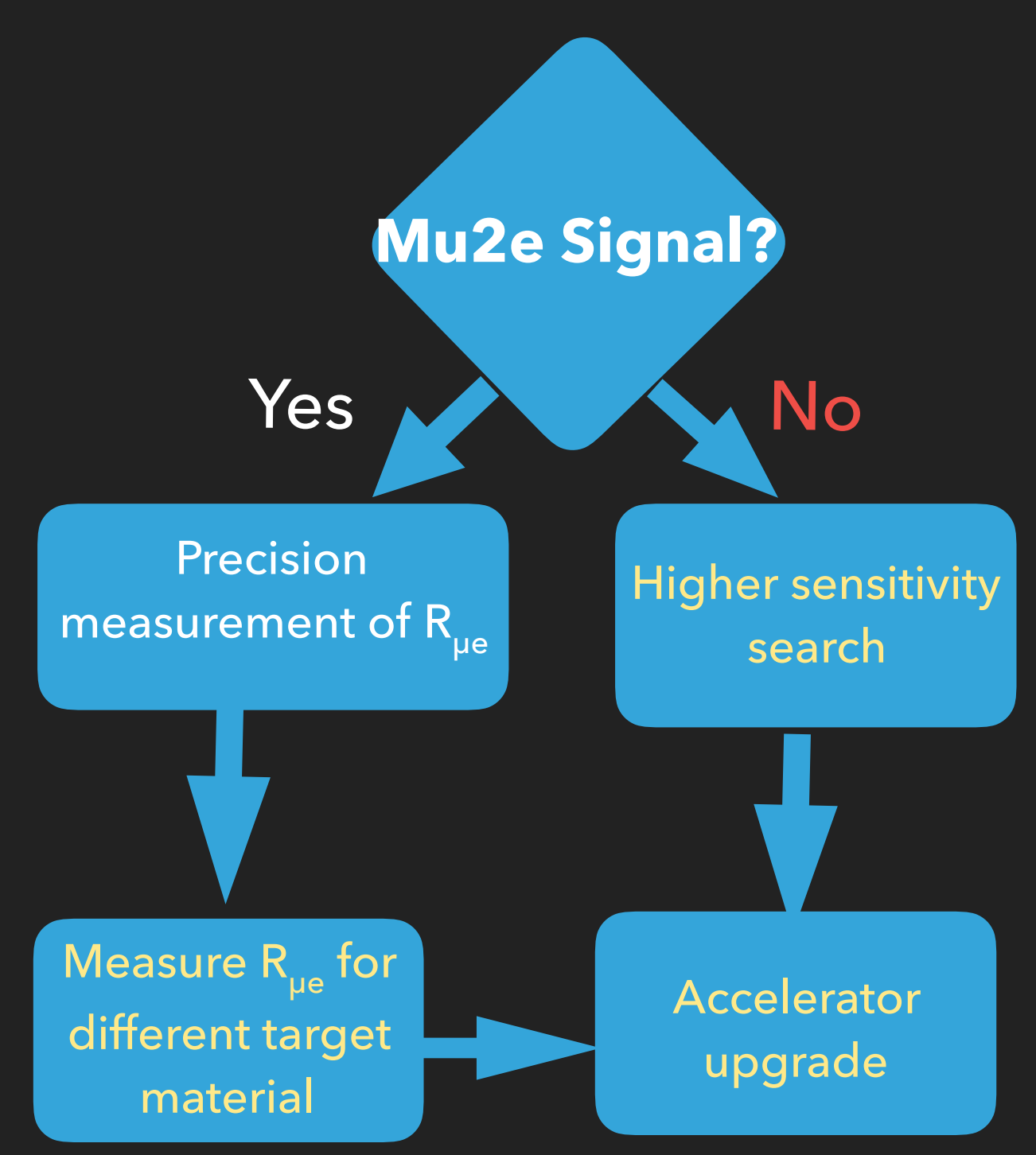

( $) R_{\mu e}<8 \times 10^{-17}$ will strongly constrain models

o Conduct next-generation search with higher sensitivity

( ) See expression of interest for Mu2e-II > arxiv.1802.02599

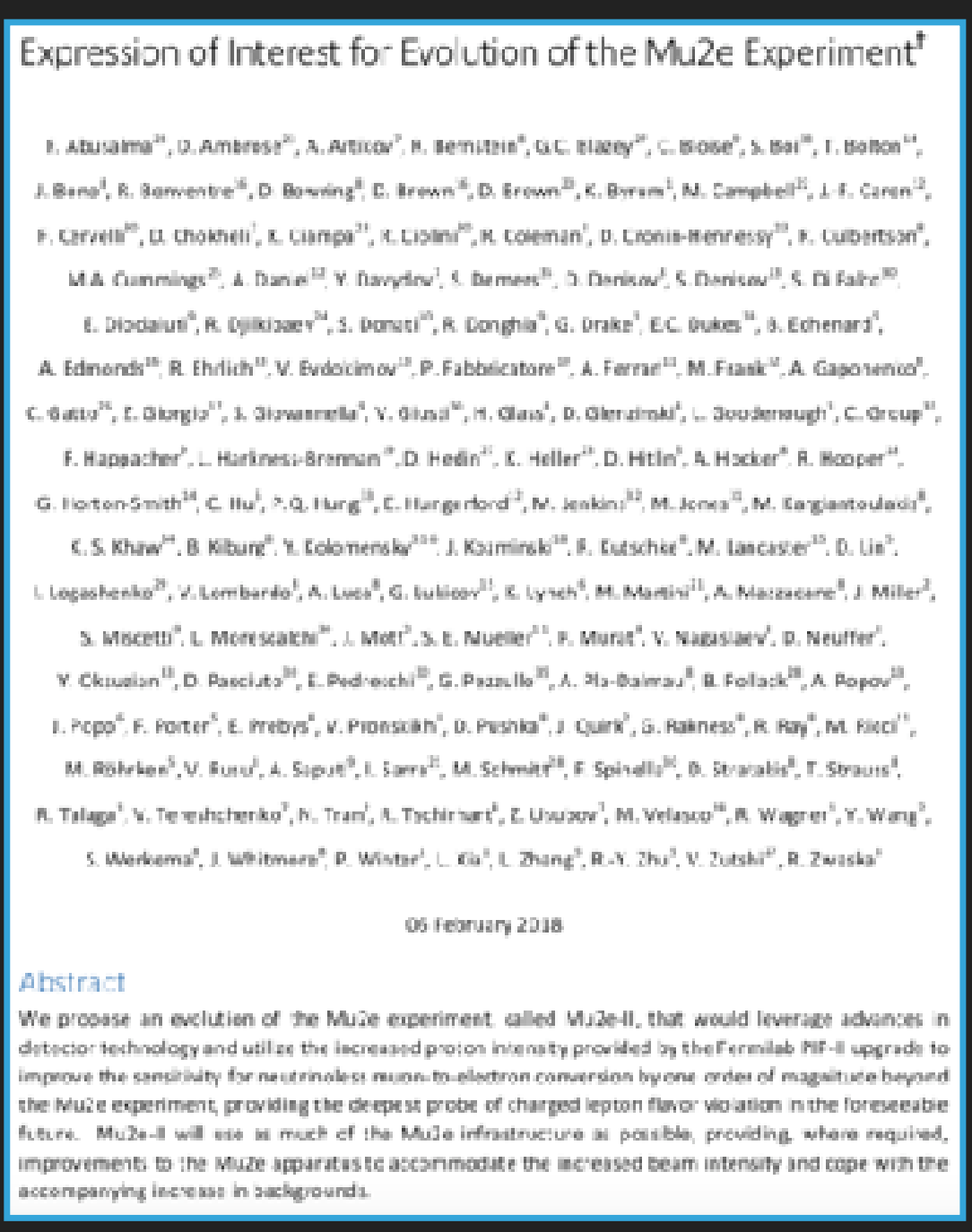




\section{Outline}

2

\section{Mu2e}
o ELFV
O Experimentat Betails
0 The Euture 
- Improve limit on $\mu N \rightarrow e N$ by four-orders of magnitude

- A signal $=$ discovery of new physics

o Many NP models predict $\mathrm{R}_{\mu \mathrm{e}} \sim$ 10-14 - 10-16

D Discovery reach $(5 \sigma): R_{\mu e} \approx 2 \times 10$-16

1. Exclusion power $(90 \% \mathrm{CL}): \mathrm{R}_{\mu \mathrm{e}} \geqq 8 \times 10^{-17}$

○ R\&D is mature, with physics data collection beginning in 2021

- Not mentioned: alternate searches are possible

- An upgraded experiment is planned

\section{Thank you!}

